\title{
LEFT-INVARIANT PARABOLIC EVOLUTIONS ON $S E(2)$ AND CONTOUR ENHANCEMENT VIA INVERTIBLE ORIENTATION SCORES PART I: LINEAR LEFT-INVARIANT DIFFUSION EQUATIONS ON $S E(2)$
}

BY

REMCO DUITS (Department of Mathematics/Computer Science and Department of Biomedical Engineering, Eindhoven University of Technology, Den Dolech 2, P.O. Box 513, 5600MB Eindhoven, The Netherlands)

AND

ERIK FRANKEN (Department of Biomedical Engineering, Eindhoven University of Technology, Den Dolech 2, P.O. Box 513, 5600MB Eindhoven, The Netherlands.)

\begin{abstract}
We provide the explicit solutions of linear, left-invariant, diffusion equations and the corresponding resolvent equations on the 2D-Euclidean motion group $S E(2)=\mathbb{R}^{2} \rtimes \mathbb{T}$. These parabolic equations are forward Kolmogorov equations for well-known stochastic processes for contour enhancement and contour completion. The solutions are given by group convolution with the corresponding Green's functions. In earlier work we have solved the forward Kolmogorov equations (or Fokker-Planck equations) for stochastic processes on contour completion. Here we mainly focus on the forward Kolmogorov equations for contour enhancement processes which do not include convection. We derive explicit formulas for the Green's functions (i.e., the heat kernels on $S E(2)$ ) of the left-invariant partial differential equations related to the contour enhancement process. By applying a contraction we approximate the left-invariant vector fields on $S E(2)$ by left-invariant generators of a Heisenberg group, and we derive suitable approximations of the Green's functions. The exact Green's functions are used in so-called collision distributions on $S E(2)$, which are the product of two left-invariant resolvent diffusions given an initial distribution on $S E(2)$. We use the left-invariant evolution processes for automated contour enhancement in noisy medical image data using
\end{abstract}

Received March 14, 2008.

2000 Mathematics Subject Classification. Primary 58J65, 49Q20, 22E30; Secondary 34B30, 37L05, $47 \mathrm{D} 06$.

Key words and phrases. Forward Kolmogorov equations, image analysis, harmonic analysis on Lie groups.

The Netherlands Organization for Scientific Research is gratefully acknowledged for financial support.

E-mail address: R.Duits@tue.nl

E-mail address: E.M.Franken@tue.nl 
a so-called orientation score, which is obtained from a grey-value image by means of a special type of unitary wavelet transformation. Here the real part of the (invertible) orientation score serves as an initial condition in the collision distribution.

1. Introduction. In many medical imaging applications, elongated structures (such as catheters, blood vessels and collagen fibres) appear only partially and vaguely in noisy medical image data, [30. It is often desirable to process these images such that crossing elongated structures become more visible before actual detection takes place. Due to occlusions, small parts of these line or edge-like structures may not be clearly visible, requiring contour completion, [47, 57, 6, 2], 20]. Furthermore, the acquisition of, for example, $X$-ray images is harmful to a patient. Therefore, the radiation dose is reduced as much as possible, which leads to very noisy images. Such images typically require contour enhancement, [30, 10, where the aim is to make the elongated structures more visible while reducing the noise. In this article we will consider operators for contour enhancement, using diffusion equations on the noncommutative group $S E(2)$ of planar translations and rotations. Rather than designing operators directly on images we first construct invertible orientation scores which are complex-valued functions on $S E(2)$, see Figures 1 and 3 , and process the image via these invertible orientation scores, see Figure 2. This approach has the practical advantage that we can handle crossing curves.

First we consider the construction of an orientation score. Image analysis usually starts with the sampling of an image $f \in \mathbb{L}_{2}\left(\mathbb{R}^{2}\right)$ by a function $\psi \in \mathbb{L}_{2}\left(\mathbb{R}^{2}\right)$ via $f \mapsto(\psi, f)_{\mathbb{L}_{2}\left(\mathbb{R}^{2}\right)}$. To probe an image at every location $\mathbf{x} \in \mathbb{R}^{2}$ and in every direction $e^{i \theta}$ one translates and rotates an anisotropic wavelet $\psi$. Here directions $e^{i \theta}$ are elements of the torus $\mathbb{T}$. This commutative group $\mathbb{T}$ is the unit sphere (the set $S^{1}$ ) in $\mathbb{C}$ equipped with the product $e^{i \theta} e^{i \theta^{\prime}}=e^{i\left(\theta+\theta^{\prime}\right)}$. The result of such an image sampling is a function $\mathcal{W}_{\psi} f \in \mathbb{L}_{2}(S E(2))$ on the Euclidean motion group manifold $S E(2)=\mathbb{R}^{2} \rtimes \mathbb{T}$, which is given by

$$
\mathcal{W}_{\psi} f(g)=\int_{\mathbb{R}^{2}} \overline{\psi\left(R_{\theta}^{-1}(\mathbf{y}-\mathbf{x})\right)} f(\mathbf{y}) \mathrm{d} \mathbf{y},
$$

where $g=\left(\mathbf{x}, e^{i \theta}\right) \in S E(2)=\mathbb{R}^{2} \rtimes \mathbb{T}, R_{\theta}=\left(\begin{array}{cc}\cos \theta & -\sin \theta \\ \sin \theta & \cos \theta\end{array}\right) \in \mathrm{SO}(2)$. Note that the mapping $f \mapsto \mathcal{W}_{\psi} f$ is a mapping from $\mathbb{L}_{2}\left(\mathbb{R}^{2}\right)$ into $\mathbb{L}_{2}(S E(2))$. Throughout this article we refer to function $\mathcal{W}_{\psi} f$ as the orientation score of image $f$; see Figure 1 .

The generation of orientation scores and the reconstruction of images thereof has been the subject of previous publications, [14, 15, 18, 37. In Section 2 and Appendix $\mathrm{A}$ we will provide an overview, containing new results on, respectively, the differences with standard wavelet theory and connections to Fourier theory on $S E(2)$. In previous work we have shown, [14, Thm. 18, App. 7.2], [12, 16, 15, that $\mathcal{W}_{\psi}$ is a unitary transformation of $\mathbb{L}_{2}\left(\mathbb{R}^{2}\right)$ onto the unique [5] reproducing kernel space $\mathbb{C}_{K}^{S E(2)}$ consisting of complex-valued functions on $S E(2)$ with reproducing kernel $K(g, h)=\left(\mathcal{U}_{g} \psi, \mathcal{U}_{h} \psi\right)_{\mathbb{L}_{2}\left(\mathbb{R}^{2}\right)}$, where

$$
\mathcal{U}_{g} \psi(\mathbf{y})=\psi\left(R_{\theta}^{-1}(\mathbf{y}-\mathbf{x})\right)
$$

for all $g=\left(\mathbf{x}, e^{i \theta}\right) \in S E(2)$. This reproducing kernel space is the space of orientation scores and equals the range of $\mathcal{W}_{\psi}$. However, in Section 2 we will show that only for a proper choice of (distributions) $\psi$ does the norm on this reproducing kernel space (of 

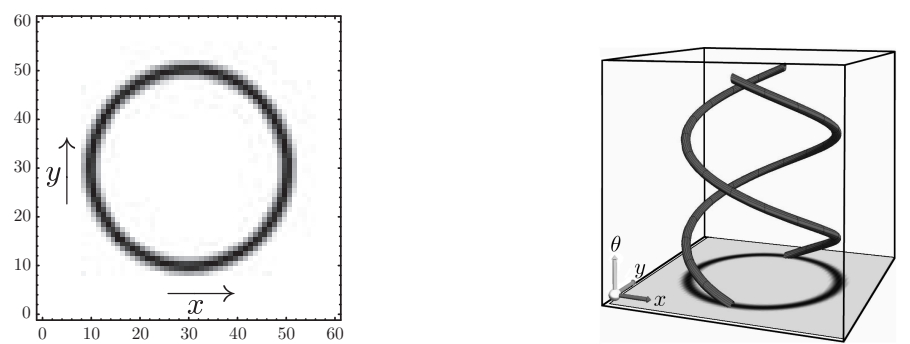

FIG. 1. Illustration of an example image (left) $f: \mathbb{R}^{2} \rightarrow \mathbb{R}$, mapping to a given position $(x, y) \in \mathbb{R}^{2}$ to a grey-value $f(x, y) \in \mathbb{R}$, and an isointensity surface (right) $\left\{\left(x, y, e^{i \theta}\right) \in S E(2):\left|\mathcal{W}_{\psi} f\left(x, y, e^{i \theta}\right)\right|=c\right\}$ of the absolute value $\left|\mathcal{W}_{\psi} f\right|$ of an orientation score $\mathcal{W}_{\psi} f: S E(2) \rightarrow$ $\mathbb{C}$, mapping an element $\left(x, y, e^{i \theta}\right) \in S E(2)$ to a complex number $\mathcal{W}_{\psi} f\left(x, y, e^{i \theta}\right) \in \mathbb{C}$. Here we have set the constant $c>0$ slightly smaller than $\max _{g \in S E(2)}\left|\mathcal{W}_{\psi} f(g)\right|$.

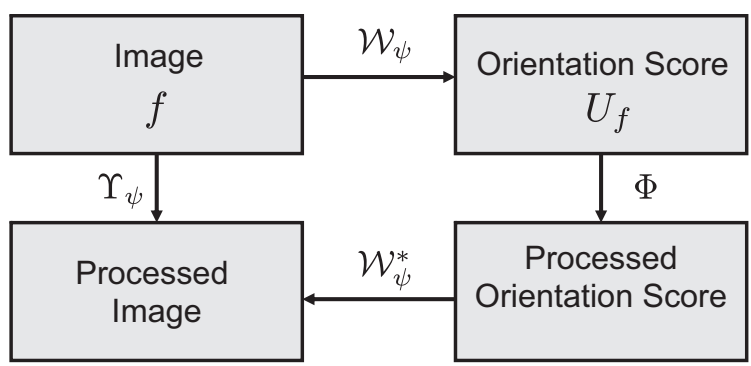

FIG. 2. A brief schematic view on image processing via invertible orientation scores. Throughout this article we shall consider suitable operators for contour enhancement based on left-invariant parabolic evolutions on $S E(2)$. Here in part I we consider operators $\Phi$ based on linear left-invariant operators, whereas in part II, 22, we shall consider $\Phi$ as a nonlinear left-invariant evolution operator.

orientation scores), for details see [14, ch:4.4, p.120], coincide with the natural restriction of the $\mathbb{L}_{2}$-norm to the closed subspace of all orientation scores.

This is important since then a small pertubation on an image corresponds to a small pertubation on its orientation score and vice versa, and consequently operators $\Phi$ on the space of orientation scores are robustly and 1-to-1 related to operators $\Upsilon_{\psi}$ on images by

$$
\Upsilon_{\psi}=\mathcal{W}_{\psi}^{*} \circ \Phi \circ \mathcal{W}_{\psi}
$$

To get a first quick impression of our scheme, see Figure 2 .

Now the wavelet transformation $\mathcal{W}_{\psi}$ between the image $f$ and the orientation score $\mathcal{W}_{\psi} f$ intertwines the left regular unitary representations $\mathcal{U}, \mathcal{L}$ of the $2 \mathrm{D}$ Euclidean motion group $S E(2)$ on, respectively, $\mathbb{L}_{2}\left(\mathbb{R}^{2}\right)$ and $\mathbb{L}_{2}(S E(2))$ and consequently, the effective operator on an image $\Upsilon_{\psi}:=\mathcal{W}_{\psi}^{*} \circ \Phi \circ \mathcal{W}_{\psi}$ is Euclidean invariant iff the corresponding operator $\Phi$ on the orientation score is left-invariant, [14, Thm. 21, p.153]. So this means 
that an operator on an orientation score must commute with left actions of the Euclidean motion group in order to obtain a Euclidean invariant operator on the image.

As a particular class of left-invariant operators we consider in section 3 all linear, second-order, left-invariant evolution equations and their resolvents on $\mathbb{L}_{2}\left(\mathbb{R}^{2} \rtimes \mathbb{T}\right)$, which correspond to the forward Kolmogorov (or Fokker-Planck) equations of left-invariant stochastic processes on the Euclidean motion group $S E(2) \equiv \mathbb{R}^{2} \rtimes \mathbb{T}$. The solutions $W(g, s)=e^{s A} W(g, 0)$ of these linear evolution equations represent the probability density of finding a random walker at position $g$ with traveling time $s>0$, given some initial distribution $g \mapsto W(g, 0)$. The solutions $P_{\alpha}(g)=\alpha(\alpha I-A)^{-1} W(g, 0)$ of the corresponding resolvent equations (obtained from $W(g, s)$ by a Laplace transform over time) represent the probability density of finding a random walker at position $g$ regardless of its traveling time, but again given the initial distribution $g \mapsto W(g, 0)$. Here the traveling time $s>0$ is memoryless and therefore negatively exponential distributed with expectation $\alpha^{-1}>0$.

We stress however that just linear left-invariant diffusions themselves (without combining them with grey-value transformations) are of no use on orientation scores, since if the operator $\Phi$ on an orientation score is linear, bounded and left-invariant, the net operator $\Upsilon_{\psi}$ on an image is an isotropic convolution. To this end we simply note that if $\Phi$ is linear and left-invariant, then $\Upsilon_{\psi}$ is linear, bounded, and Euclidean invariant. So by the Dunford-Pettis theorem, [11], $\Upsilon_{\psi}$ is a translation rotation-invariant kernel operator, which must be a convolution with an isotropic kernel, [17, and in such a trivial case, orientation scores are not needed. Instead of considering linear left-invariant diffusions we consider direct products of linear left-invariant evolution equations which are collision distributions of two linear stochastic processes. So these nonlinear operators on orientation scores boil down to solving linear, left-invariant, evolution equations. We distinguish between two types of stochastic processes on the Euclidean motion group:

(1) Stochastic processes for contour completion, including the direction process as proposed by Mumford [47.

(2) Stochastic processes for contour enhancement, including the cortical model of the visual system for contour enhancement as proposed by Citti et al. [10.

The mathematical difference between these two types of stochastic processes is that the generator of the forward Kolmogorov equation of stochastic processes of the first type, in contrast to the forward Kolmogorov equations of stochastic processes of the second type, contains a convection part that fills and bridges gaps in lines and contours; see 20], Fig. 1, Ch: 1 for two typical examples.

The intuitive difference between contour enhancement and completion in image analysis is that contour enhancement aims for robust de-noising of elongated structures as a pre-processing step for detection of elongated structures in noisy images, whereas contour completion aims for completion of interrupted curves due to, for example, occlusion or due to the application of thresholds on grey-levels. The difference between enhancement and completion will clearly be reflected in both the trajectories of the random walks in the underlying stochastic processes, Figure 5 , and in the shape of iso-contours of the Green's functions, Figures 7 and 9 , 
In this article we mainly consider linear and nonlinear stochastic processes for contour enhancement, in contrast to our related earlier work [20, where we considered stochastic processes for contour completion, 20, 2, 14. Occasionally, we will briefly return to the contour completion process to stress the analogy and differences between (the solutions of) the forward Kolmogorov equations of the contour completion and contour enhancement processes. In comparison to 20, we will provide much more detailed information in Section 2 on the framework of invertible orientation scores, including new results on both the embedding in standard wavelet theory and Fourier theory on $S E(2)$ in Appendix A. Section 2 serves as an essential prerequisite for contour enhancement in images, which we consider in Sections 3. 4. Here we put much more emphasis on both the underlying (discrete) stochastic processes and the Hörmander condition. Finally, we present a better connection (compared to our earlier work on contour completion [20]) of the exact solutions to the Heisenberg approximations [20] by means of contraction, [52].

In Section 4 we restrict ourselves to direct products of two linear left-invariant resolvent diffusions on $S E(2)$ as suitable operators for contour enhancement on invertible orientation scores. These direct products are the probability density of collision of oriented grey-value particles moving from a source distribution, with oriented grey-value particles of a sink distribution. In the context of contour completion this is a well-known technique in image analysis, [59. In this article, however, we apply this technique to contour enhancement and restrict ourselves to the case where both the sink and source distribution are equal to the real part of an orientation score of the input image. Although the nonlinear adaptive left-invariant diffusion equations, which we discuss in part II of our article, applied to invertible orientation scores seem to lead to visually more appealing results of enhancing elongated structures in noisy medical image data, we consider these products of linear evolutions for three reasons. Firstly, they are easier to implement, secondly they involve fewer parameters, and thirdly they are much easier to analyse.

The solutions of the linear left-invariant evolution equations are given by $S E(2)$ convolution with the corresponding Green's function. As explicit formulae for the Green's functions for contour enhancement (i.e., the heat kernels on $S E(2)$ ) were missing in our earlier work [20, we explicitly derive them in Section [5. Here we follow two approaches, comparable to our two approaches in [20, where we derived the exact Green's functions of Mumford's direction process, [47. Both approaches are described in Subsection 5.1 .

Then in Subsection 5.2 we approximate (analogously to [20, ch: 4.3]) the left-invariant basis of the Euclidean group generators by left-invariant generators of a Heisenberg-type group. The resulting equations render simple, analytic approximations, which do not exactly coincide with the closely related approximations derived in [10.

In Subsection 5.3 we apply the results by Hörmander [36] and provide stochastic insight in the induced smoothing in the "missing" directions in the diffusion processes on $S E(2)$ generated by hypo-elliptic (not elliptic) operators. We also explain why the singular behavior that occurred in the Heisenberg approximation of the Green's function of the contour completion [20, ch: 4.3] does not occur in the contour-enhancement case. 
Finally, in Section 5.4 we also provide Gaussian estimates and new practical, accurate, asymptotic formulas for both the exact and the approximative Green's functions of the contour-enhancement process in Subsection 5.4 .

2. Invertible orientation scores. In many image analysis applications a function $U_{f} \in \mathbb{L}_{2}(S E(2))$ defined on the $2 \mathrm{D}$ Euclidean motion group $S E(2)=\mathbb{R}^{2} \rtimes \mathbb{T}$ is constructed from a $2 \mathrm{D}$ grey-value image $f \in \mathbb{L}_{2}\left(\mathbb{R}^{2}\right)$. Such a function is supposed to provide an overview of all local orientations in an image. This is important for perceptual organization, [37, 28, 44, 18, 15, 56, 7, and is inspired by the visual system of mammals, in which receptive fields exist that are tuned to various locations and orientations, 8]. In addition to the approach given in the introduction there exist many other ways, [6, 24, 28, 44] and [14, Ch.5], to construct a function $U_{f}: \mathbb{R}^{2} \rtimes \mathbb{T} \rightarrow \mathbb{C}$ from an image $f: \mathbb{R}^{2} \rightarrow \mathbb{R}$, but usually these approaches do not consider the stability of the inverse transformation $U_{f} \mapsto f$.

In this section we consider the case $U_{f}=\mathcal{W}_{\psi} f$, as is given in the introduction (1.1). This case leads to the framework of invertible orientation scores, which we developed in previous work, $14,18,15$, and which we summarize here. Moreover, we will provide a better view on our previous results in Appendix $\mathrm{A}$.

An orientation score $\mathcal{W}_{\psi} f: \mathbb{R}^{2} \rtimes \mathbb{T} \rightarrow \mathbb{C}$ of an image $f: \mathbb{R}^{2} \rightarrow \mathbb{R}$ is obtained by correlation with an anisotropic kernel $\psi: \mathbb{R}^{2} \rightarrow \mathbb{C}$ via (1.1). Assume $\psi \in \mathbb{L}_{2}\left(\mathbb{R}^{2}\right) \cap \mathbb{L}_{1}\left(\mathbb{R}^{2}\right)$. Then the transform $\mathcal{W}_{\psi}$, cf. (1.1), which maps image $f \in \mathbb{L}_{2}\left(\mathbb{R}^{2}\right)$ onto its orientation score $\mathcal{W}_{\psi} f \in \mathbb{L}_{2}\left(\mathbb{R}^{2} \rtimes \mathbb{T}\right)$ can be rewritten, in inner product form, as

$$
\left(\mathcal{W}_{\psi} f\right)(g)=\left(\mathcal{U}_{g} \psi, f\right)_{\mathbb{L}_{2}\left(\mathbb{R}^{2}\right)},
$$

where $g \mapsto \mathcal{U}_{g}$ is a unitary (group) representation of the Euclidean motion group $S E(2)=$ $\mathbb{R}^{2} \rtimes \mathbb{T}$ into $\mathbb{L}_{2}\left(\mathbb{R}^{2}\right)$ given by (1.2). Note that the representation $\mathcal{U}$ is reducible as it leaves the following closed subspaces invariant: $\left\{f \in \mathbb{L}_{2}\left(\mathbb{R}^{2}\right) \mid \operatorname{supp}\{\mathcal{F}[f]\} \subset B_{\mathbf{0}, \varrho}\right\}$, $\varrho>0$, where $B_{\mathbf{0}, \varrho}$ denotes the ball with center $\mathbf{0} \in \mathbb{R}^{2}$ and radius $\varrho>0$ and where $\mathcal{F}: \mathbb{L}_{2}\left(\mathbb{R}^{2}\right) \rightarrow \mathbb{L}_{2}\left(\mathbb{R}^{2}\right)$ denotes the Fourier transform given by

$$
\mathcal{F} f(\boldsymbol{\omega})=\frac{1}{2 \pi} \int_{\mathbb{R}^{2}} f(\mathbf{x}) e^{-i \boldsymbol{\omega} \cdot \mathbf{x}} \mathrm{d} \mathbf{x},
$$

for almost every $\boldsymbol{\omega} \in \mathbb{R}^{2}$ and all $f \in \mathbb{L}_{2}\left(\mathbb{R}^{2}\right)$. This differs from standard continuous wavelet theory; see, for example, 39 and [4], where the wavelet transform is constructed by means of a quasi-regular representation of the similitude group $\operatorname{SIM}(2)=\mathbb{R}^{2} \rtimes \mathbb{T} \times \mathbb{R}^{+}$, which is unitary, irreducible and square integrable (admitting the application of the more general results in [33]). For image analysis this means that we do allow a stable reconstruction already at a single scale orientation score for a proper choice of $\psi$. In standard continuous wavelet reconstruction schemes with $\psi \in \mathbb{L}_{2}\left(\mathbb{R}^{2}\right) \cap \mathbb{L}_{1}\left(\mathbb{R}^{2}\right)$ and

$$
W_{\psi} f\left(\mathbf{x}, e^{i \theta}, a\right)=\left(\mathcal{V}_{\left(\mathbf{x}, e^{i \theta}, a\right)} \psi, f\right)_{\mathbb{L}_{2}\left(\mathbb{R}^{2}\right)},
$$

with irreducible representation $\mathcal{V}: S I M(2) \rightarrow \mathcal{B}\left(\mathbb{L}_{2}\left(\mathbb{R}^{2}\right)\right)$ given by

$$
\mathcal{V}_{\left(\mathbf{x}, e^{i \theta}, a\right)} \psi(\mathbf{y})=\frac{1}{a} \psi\left(a^{-1} R_{\theta}^{-1}(\mathbf{y}-\mathbf{x})\right),
$$


however, it is not possible to obtain an image $f$ in a well-posed manner from a "fixed scale layer", that is, from $\mathcal{W}_{\psi} f(\cdot, \cdot, a) \in \mathbb{L}_{2}\left(\mathbb{R}^{2} \rtimes \mathbb{T}\right)$, for fixed scale $a>0$.

This shortcoming in the standard wavelet theoretical framework directly follows from the fact that the standard necessary and sufficient wavelet admissibility condition

$$
C_{\psi}=\frac{1}{(\psi, \psi)} \int_{S I M(2)}\left|\left(\mathcal{V}_{g} \psi, \psi\right)\right|^{2} \mathrm{~d} \mu_{\operatorname{SIM}(2)}(g)<\infty
$$

where $\mathrm{d} \mu_{S I M(2)}$ denotes the left-invariant Haar measure on $\operatorname{SIM}(2)$, allowing stable reconstruction of $f$ from $W_{\psi} f \in \mathbb{L}_{2}(S I M(2))$ via the $\mathbb{L}_{2}$-adjoint (i.e., $f=W_{\psi}^{*} W_{\psi} f$ ) conflicts with the necessary and sufficient condition, [14, 31,

$$
M_{\mathcal{D}_{a} \psi}(\boldsymbol{\omega}):=\int_{S O(2)}\left|\mathcal{F} \psi\left(a R_{\theta}^{T} \boldsymbol{\omega}\right)\right|^{2} \mathrm{~d} \theta=1 \text { for all } \boldsymbol{\omega} \in \mathbb{R}^{2},
$$

for some fixed $a>0$ for stable reconstruction of $f$ from $W_{\psi} f(\cdot, \cdot, a) \in \mathbb{L}_{2}(S E(2))$ via the adjoint $f=\left(W_{\psi}(\cdot, \cdot, a)\right)^{*} W_{\psi} f(\cdot, \cdot, a)$. Here the unitary dilation operator $\mathcal{D}_{a}$ is given by $\mathcal{D}_{a} \psi(\mathbf{x})=a^{-1} \psi\left(a^{-1} \mathbf{x}\right), a>0, \mathbf{x} \in \mathbb{R}^{2}, \psi \in \mathbb{L}_{2}\left(\mathbb{R}^{2}\right)$.

To this end we note that by a brief and well-known calculation (for details see, for example, 41, pp. 52, 53]) equality (2.4) is equivalent to

$$
C_{\psi}=4 \pi^{2} \int_{\mathbb{R}^{2}} \frac{|\mathcal{F} \psi(\boldsymbol{\omega})|^{2}}{\|\boldsymbol{\omega}\|^{2}} \mathrm{~d} \boldsymbol{\omega}<\infty
$$

so that it implies $\mathcal{F} \psi(\mathbf{0})=0$ and thereby the continuous function $M_{\psi}$ vanishes at $\mathbf{0}$. So clearly, condition (2.5) cannot be satisfied 1 See Example 2.1.

Moreover, the general wavelet reconstruction results [33] do not apply to the transform $f \mapsto \mathcal{W}_{\psi} f$, since our representation $\mathcal{U}$ is reducible. In earlier work we therefore provided a general theory [14, 12, to construct wavelet transforms associated with admissible vectors/ distributions 2 With these wavelet transforms we construct orientation scores $\mathcal{W}_{\psi} f: \mathbb{R}^{2} \rtimes \mathbb{T} \rightarrow \mathbb{C}$ by means of admissible line detecting wavelet: $3 \psi \in \mathbb{L}_{2}\left(\mathbb{R}^{2}\right)$ such that the transform $\mathcal{W}_{\psi}$ is unitary onto the unique reproducing kernel Hilbert space $\mathbb{C}_{K}^{S E(2)}$ of functions on $S E(2)$ with reproducing kernel $K(g, h)=\left(\mathcal{U}_{g} \psi, \mathcal{U}_{h} \psi\right)$, which is a closed vector subspace of $\mathbb{L}_{2}(S E(2))$. For the abstract construction of the unique reproducing kernel space $\mathbb{C}_{K}^{\mathbb{I}}$ on a set $\mathbb{I}$ (not necessarily a group) from a function of positive type $K: \mathbb{I} \times \mathbb{I} \rightarrow \mathbb{C}$, we refer to the early work of Aronszajn [5]. Here we only provide the essential Plancherel formula, which can also be found in a slightly different way in the work of Führ 31, for the wavelet transform $\mathcal{W}_{\psi}$ and which provides a more tangible description of the norm on $\mathbb{C}_{K}^{S E(2)}$ rather than the abstract one in 5 . To this end we note that we can write

$$
\left(\mathcal{W}_{\psi} f\right)\left(\mathbf{x}, e^{i \theta}\right)=\left(\mathcal{U}_{\left(\mathbf{x}, e^{i \theta}\right)} \psi, f\right)_{\mathbb{L}_{2}\left(\mathbb{R}^{2}\right)}=\left(\mathcal{F} \mathcal{T}_{\mathbf{x}} \mathcal{R}_{\theta} \psi, \mathcal{F} f\right)_{\mathbb{L}_{2}\left(\mathbb{R}^{2}\right)}=\mathcal{F}^{-1}\left(\overline{\mathcal{R}_{\theta} \mathcal{F} \psi} \cdot \mathcal{F} f\right)(\mathbf{x}),
$$

\footnotetext{
${ }^{1}$ For well-posed reconstruction it is not necessary to have reconstruction by the $\mathbb{L}_{2}$-adjoint. In principle for $M_{\psi}$ bounded from below and above one may use the inverse (2.8) (which is the adjoint if we impose the reproducing kernel norm on orientation scores, 14 pp. 123-124, Thm. 19]), but even then the inverse (2.8) is ill-posed as $M_{\psi}$ is not globally bounded from below.

${ }^{2}$ Depending on whether images are assumed to be band-limited or not; for full details, see 13.

${ }^{3}$ Or rather admissible distributions $\psi \in \mathbb{H}^{-(1+\epsilon), 2}\left(\mathbb{R}^{2}\right), \epsilon>0$ if one does not want a restriction to band-limited images.
} 
where the rotation and translation operators on $\mathbb{L}_{2}\left(\mathbb{R}^{2}\right)$ are defined by $\mathcal{R}_{\theta} f(\mathbf{y})=f\left(R_{\theta}^{-1} \mathbf{y}\right)$ and $\mathcal{T}_{\mathbf{x}} f(\mathbf{y})=f(\mathbf{y}-\mathbf{x})$. Consequently, we find that

$$
\begin{aligned}
\left\|\mathcal{W}_{\psi} f\right\|_{\mathbb{C}_{K}^{S E(2)}}^{2} & =\int_{\mathbb{R}^{2}} \int_{\mathbb{T}}\left|\left(\mathcal{F} \mathcal{W}_{\psi} f\right)\left(\boldsymbol{\omega}, e^{i \theta}\right)\right|^{2} \mathrm{~d} \theta \frac{1}{M_{\psi}(\boldsymbol{\omega})} \mathrm{d} \boldsymbol{\omega} \\
& =\int_{\mathbb{R}^{2}} \int_{\mathbb{T}}|(\mathcal{F} f)(\boldsymbol{\omega})|^{2}\left|\mathcal{F} \psi\left(R_{\theta}^{T} \boldsymbol{\omega}\right)\right|^{2} \mathrm{~d} \theta \frac{1}{M_{\psi}(\boldsymbol{\omega})} \mathrm{d} \boldsymbol{\omega} \\
& =\int_{\mathbb{R}^{2}}|(\mathcal{F} f)(\boldsymbol{\omega})|^{2} \mathrm{~d} \boldsymbol{\omega}=\|f\|_{\mathbb{L}_{2}\left(\mathbb{R}^{2}\right)}^{2},
\end{aligned}
$$

where $M_{\psi} \in C\left(\mathbb{R}^{2}, \mathbb{R}\right)$ is defined by

$$
M_{\psi}(\boldsymbol{\omega}):=\int_{0}^{2 \pi}\left|\mathcal{F} \psi\left(R_{\theta}^{T} \boldsymbol{\omega}\right)\right|^{2} \mathrm{~d} \theta .
$$

If $\psi$ is chosen such that $M_{\psi}=1$, then by (2.6) we gain $\mathbb{L}_{2}$-norm preservation. However, this is not possible as $\psi \in \mathbb{L}_{2}\left(\mathbb{R}^{2}\right) \cap \mathbb{L}_{1}\left(\mathbb{R}^{2}\right)$ implies that $M_{\psi}$ is a continuous function vanishing at infinity. Now, theoretically speaking, one can use a Gelfand-triple structure generated by $\sqrt{1+|\Delta|}$ to allow distributional wavelets $\psi \in \mathbb{H}^{-k}\left(\mathbb{R}^{2}\right), k>1$ with the property $M_{\psi}=1$, so that $\psi$ has equal length in each irreducible subspace (which uniquely corresponds to the dual orbits of $S O(2)$ on $\mathbb{R}^{2}$ ); for details see Appendix $\mathrm{A}$ and for generalizations see [13. In practice, however, because of finite grid sampling, we can as well restrict $\mathcal{U}$ (which is well-defined) to the space of band-limited images.

Finally, since the wavelet transform $\mathcal{W}_{\psi}$ maps the space of images $\mathbb{L}_{2}\left(\mathbb{R}^{2}\right)$ unitarily onto the space of orientation scores $\mathbb{C}_{K}^{S E(2)}$ (provided that $M_{\psi}>0$ ), [14, Thm. 18], we can reconstruct the original image $f: \mathbb{R}^{2} \rightarrow \mathbb{R}$ from its orientation score $\mathcal{W}_{\psi} f: S E(2) \rightarrow \mathbb{C}$ by means of the adjoint

$$
f=\mathcal{W}_{\psi}^{*} \mathcal{W}_{\psi}[f]=\mathcal{F}^{-1}\left[\boldsymbol{\omega} \mapsto \int_{0}^{2 \pi} \mathcal{F}\left[\mathcal{W}_{\psi} f\left(\cdot, e^{i \theta}\right)\right](\boldsymbol{\omega}) \mathcal{F}\left[\mathcal{R}_{e^{i \theta}} \psi\right](\boldsymbol{\omega}) \mathrm{d} \theta M_{\psi}^{-1}(\boldsymbol{\omega})\right] .
$$

For typical examples (and different classes) of wavelets $\psi$ such that $M_{\psi}=1$ and for details on fast approximative reconstructions, see [29, 15, 16]. For an illustration of a typical proper wavelet $\psi$ (i.e., $M_{\psi} \approx 1$ ) with corresponding transformation $\mathcal{W}_{\psi} f$ and corresponding $M_{\psi}: \mathbb{R}^{2} \rightarrow \mathbb{R}^{+}$, see Figure 3 .

With this well-posed unitary transformation between the space of images and the space of orientation scores at hand, we can perform image processing via orientation scores; see [15, 16, 18, 37. For the remainder of the article we assume that $\mathcal{W}_{\psi} f$ is some given function in $\mathbb{L}_{2}(S E(2))$ and we write $\mathcal{W}_{\psi} f \in \mathbb{L}_{2}(S E(2))$ rather than $\mathcal{W}_{\psi} f \in \mathbb{C}_{K}^{S E(2)}$.

\footnotetext{
${ }^{4}$ To be precise: According to [5], 46], the norm on the space $\mathbb{C}_{K}^{S E(2)}$ with reproducing kernel $K(g, h)=\left(\mathcal{U}_{g} \psi, \mathcal{U}_{h} \psi\right)$ is given by

$\|U\|_{\mathbb{C}_{K}^{S E(2)}}^{2}=\sup \left\{\left|\sum_{j=1}^{l} \alpha_{j} \overline{U\left(g_{j}\right)}\right|^{2}\left(\sum_{k, j=1}^{l} \overline{\alpha_{k}} \alpha_{j} K\left(g_{k}, g_{j}\right)\right)^{-1} \mid l \in \mathbb{N}, \alpha_{j} \in \mathbb{C}, g_{j} \in S E(2), \sum_{k, j=1}^{l} \overline{\alpha_{k}} \alpha_{j} K\left(g_{k}, g_{j}\right) \neq 0\right\}$.

Now according to our previous more general results in [14, Thm. 18, appendix 7.2], [12], $\mathcal{W}_{\psi}$ : $\mathbb{L}_{2}\left(\mathbb{R}^{2}\right) \rightarrow \mathbb{C}_{K}^{S E(2)}$ is unitary. Now by (2.6) a more tangible description of the same norm is given by $\|U\|_{\mathbb{C}_{K}^{S E(2)}}^{2}=\int_{\mathbb{R}^{2}} \int_{\mathbb{T}}\left|\mathcal{F}_{\mathbb{R}^{2}} U(\cdot, \theta)(\omega)\right|^{2} \mathrm{~d} \theta M_{\psi}(\omega)^{-1} \mathrm{~d} \omega$. This norm equals the $\mathbb{L}_{2}(S E(2))$ norm iff $M_{\psi}=1$.
} 
EXAmple 2.1. Consider $\psi(x, y)=\check{\psi}(-x,-y)$, with

$$
\check{\psi}(x, y)=\frac{1}{\sqrt{\pi}} z e^{-\frac{|z|^{2}}{2}}=\frac{1}{\sqrt{\pi}} 2 \partial_{z} e^{-\frac{|z|^{2}}{2}}=\frac{1}{\sqrt{\pi}}\left(\partial_{x}-i \partial_{y}\right) e^{-\frac{x^{2}+y^{2}}{2}}, \quad z=x+i y .
$$

A direct computation yields $C_{\psi}=4 \pi^{2}$, so $\psi$ is admissible in the classical sense. Then according to 33 , the reconstruction is given by

$$
\begin{aligned}
f=W_{\psi}^{*} W_{\psi} f & =\frac{1}{C_{\psi}} \int_{-\pi}^{\pi} \int_{\mathbb{R}^{+}} \int_{\mathbb{R}}\left(W_{\psi} f\right)\left(b, a, e^{i \theta}\right) U_{b, a, \theta} \psi\left(2 \pi a^{3}\right)^{-1} \mathrm{~d} b \mathrm{~d} a \mathrm{~d} \theta \\
& =\frac{1}{4 \pi^{2}} \int_{0}^{\infty} \int_{-\pi}^{\pi} f * \overline{\mathcal{D}_{a} \mathcal{R}_{\theta} \tilde{\psi}} * \mathcal{D}_{a} \mathcal{R}_{\theta} \psi\left(2 \pi a^{3}\right)^{-1} \mathrm{~d} a \mathrm{~d} \theta .
\end{aligned}
$$

However, a reconstruction from a single scale layer, that is, a reconstruction of $f$ from $\mathcal{W}_{\psi} f(a, \cdot, \cdot)$ by means of (2.8) is extremely ill-posed as we have $M_{\mathcal{D}_{a} \psi}(\omega)=\rho^{2} e^{-(a \rho)^{2}}$, for $a>0$ fixed. This example (2.9) is special since the solution of the following diffusion problem (in image analysis known as Gaussian scale space, [49, 38, 26, 17]):

$$
\left\{\begin{array}{l}
\partial_{s} u(x, y, s)=\Delta_{x, y} u(x, y, s)=\left(\left(\partial_{x}^{2}+\partial_{y}^{2}\right) u\right)(x, y, s), \quad(x, y) \in \mathbb{R}^{2}, s>0 \\
u(x, y, 0)=f(x, y)
\end{array}\right.
$$

is given by $u(x, y, s)=\left(G_{s} * f\right)(x, y)$. Now set scale $s=a^{2}>0$. Then it respectively follows by $\Delta=4 \partial_{z} \partial_{\bar{z}}, G_{\frac{s}{2}} * G_{\frac{s}{2}}=G_{s}, \partial_{s} G_{s}=\Delta G_{s}$ and $\mathcal{R}_{\theta} \check{\psi}=e^{i \theta} \check{\psi}$ that the wavelet reconstruction formula (2.10) simply coincides with integration after differentiation of the semigroup generated by the heat kernel:

$$
\begin{aligned}
f & =-\int_{0}^{\infty} \partial_{s}\left(f * G_{s}\right) \mathrm{d} s=-\int_{0}^{\infty}\left(f * \Delta G_{s}\right) \mathrm{d} s=-4 \int_{0}^{\infty} f * \partial_{\bar{z}} \partial_{z} G_{s} \mathrm{~d} s \\
& =-2 \int_{0}^{\infty} f * \overline{2 \partial_{z} G_{\frac{a^{2}}{2}}} * 2 \partial_{z} G_{\frac{a^{2}}{2}} a \mathrm{~d} a \\
& =\frac{1}{4 \pi^{2}} \int_{0}^{\infty} \int_{-\pi}^{\pi} f * \overline{\mathcal{D}_{a} \mathcal{R}_{\theta} \check{\psi}} * \mathcal{D}_{a} \mathcal{R}_{\theta} \psi\left(2 \pi a^{3}\right)^{-1} \mathrm{~d} a \mathrm{~d} \theta=W_{\psi}^{*} W_{\psi} f .
\end{aligned}
$$

Also in scale space theory a reconstruction of $f$ from $u_{f}(\cdot, s)$ (i.e., inverse diffusion), $s>0$, is clearly ill-posed. A first alternative, as proposed in [37, to (2.9), which does allow well-posed single scale reconstruction, is given by the pointwise limit:

$$
\psi_{a}(x, y)=\frac{1}{a} \lim _{N \rightarrow \infty} \sum_{n=0}^{N}\left(\frac{z}{a}\right)^{n} \frac{e^{-\frac{|z|^{2}}{a^{2}}}}{\sqrt{n !}},
$$

since it satisfies $M_{\psi_{a}}=1$ for all $a>0$. But even this choice (2.12) has serious practical disadvantages (for details, see [14 pp. 141-142, App. 7.3, pp. 222-224]) compared to the other class of proper wavelets discussed in [14, ch: 4.6.1, pp. 131-136], which we used in the experiments within this paper; see Figure 3 and Figure 9.

3. Left-invariant evolution equations on the Euclidean motion group. The group product within the group $S E(2)$ of planar translations and rotations is

$$
g g^{\prime}=\left(\mathbf{x}, e^{i \theta}\right)\left(\mathbf{x}^{\prime}, e^{i \theta^{\prime}}\right)=\left(\mathbf{x}+R_{\theta} \mathbf{x}^{\prime}, e^{i\left(\theta+\theta^{\prime}\right)}\right), \quad g=\left(\mathbf{x}, e^{i \theta}\right), g^{\prime}=\left(\mathbf{x}^{\prime}, e^{i \theta^{\prime}}\right) \in S E(2),
$$

with $R_{\theta}=\left(\begin{array}{cc}\cos \theta & -\sin \theta \\ \sin \theta & \cos \theta\end{array}\right) \in S O(2)$. The tangent space at the unity element $e=\left(0,0, e^{i 0}\right)$, $T_{e}(S E(2))$, is a $3 \mathrm{D}$ Lie algebra equipped with Lie product $[A, B]=$ 
(a)

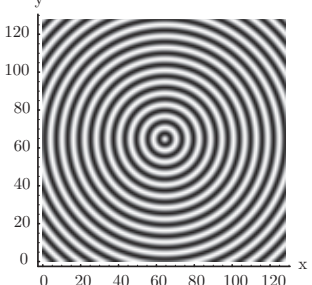

(e)

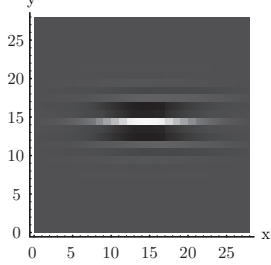

(b)

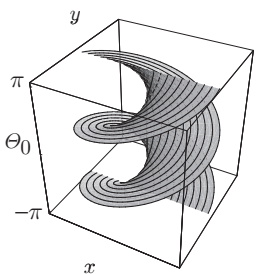

(f)

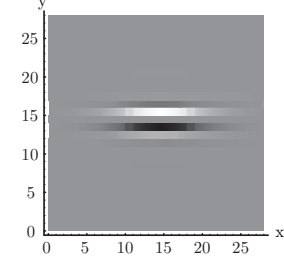

(c)
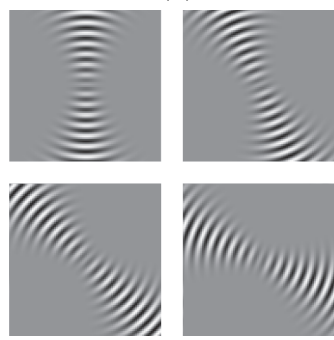

(g)

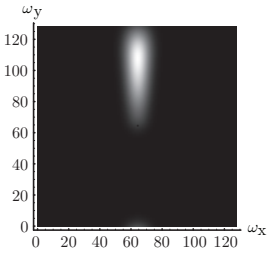

(d)

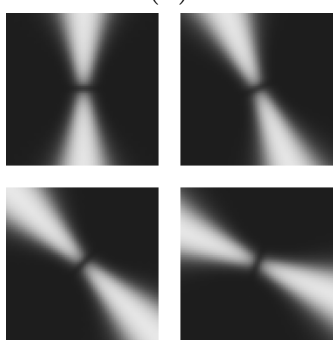

(h)

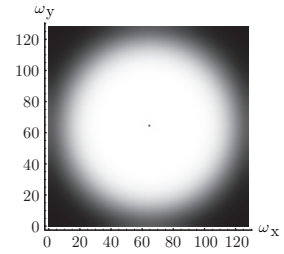

Fig. 3. (a) Example image $(x, y) \mapsto f(x, y)$. (b) The structure of the domain of the corresponding orientation score $\mathcal{W}_{\psi}[f]$. The lifted circles in the example image become spirals and all spirals are situated in the same helicoid-shaped surface. The absolute value of an orientation score $\left|\mathcal{W}_{\psi} f\right|$ is mainly concentrated around this surface, (i.e., $\left|\mathcal{W}_{\psi} f\right|$ attains high values in the direct neighborhood of this surface). (c) Real part of orientation score $(x, y) \mapsto \mathcal{W}_{\psi} f\left(x, y, e^{i \theta}\right)$ displayed for 4 different fixed orientations. (d) The absolute value $(x, y) \mapsto\left|\mathcal{W}_{\psi} f\left(x, y, e^{i \theta}\right)\right|$ yields a phase-invariant and positive response displayed for 4 fixed orientations. (e) Real part of the wavelet $\psi(\mathbf{x})=\frac{e^{-\frac{\|\mathbf{x}\|^{2}}{4 s}}}{\sqrt{4 \pi s}} \mathcal{F}^{-1}\left[\boldsymbol{\omega} \mapsto B^{k}\left(\frac{n_{\theta}\left((\phi \bmod 2 \pi)-\frac{\pi}{2}\right)}{2 \pi}\right) \mathcal{M}(\rho)\right](\mathbf{x})$, where $\mathcal{M}(\rho)=\frac{e^{-\frac{\rho^{2}}{2 \sigma^{2}}}}{\sum_{k=0}^{q}(-1)^{k}\left(2^{-1} \sigma^{-2} \rho^{2}\right)^{k}}$, with $\sigma=\frac{\varrho}{2}$ and Nyquist frequency $\varrho$ and $k$-th order $B$-spline $B_{k}=B_{0} *^{k} B_{0}$ and $B_{0}(x)=1_{\left[-\frac{1}{2}, \frac{1}{2}\right]}(x)$ and parameter values $k=2, q=4, \frac{1}{2} \sigma^{2}=400, s=10, n_{\theta}=64$. (f) Imaginary part of $\psi$. (g) The function $|\mathcal{F} \psi|^{2}$ (h) The function $M_{\psi}$. In all images grey-values have been scaled to $[0,1]$, where 0 is black and 1 is white.

$\lim _{t \downarrow 0} t^{-2}\left(a(t) b(t)(a(t))^{-1}(b(t))^{-1}-e\right)$, where $t \mapsto a(t)$, resp. $t \mapsto b(t)$, are any smooth curves in $G$ with $a(0)=b(0)=e$ and $a^{\prime}(0)=A$ and $b^{\prime}(0)=B$. Define $\left\{A_{1}, A_{2}, A_{3}\right\}:=$ $\left\{\mathbf{e}_{\theta}, \mathbf{e}_{x}, \mathbf{e}_{y}\right\}$. Then $\left\{A_{1}, A_{2}, A_{3}\right\}$ form a basis of $T_{e}(S E(2))$ and their Lie products are

$$
\left[A_{1}, A_{2}\right]=A_{3}, \quad\left[A_{1}, A_{3}\right]=-A_{2}, \quad\left[A_{2}, A_{3}\right]=0 .
$$

A vector field on $S E(2)$ (considered as a differential operator) is called left-invariant if for all $g \in G$ the push-forward of $\left(L_{g}\right)_{*} X_{e}$ by left multiplication $L_{g} h=g h$ equals $X_{g}$; i.e.,

$$
\left(X_{g}\right)=\left(L_{g}\right)_{*}\left(X_{e}\right) \Leftrightarrow X_{g} f=X_{e}\left(f \circ L_{g}\right) \text {, for all } f \in C^{\infty}: \Omega_{g} \rightarrow \mathbb{R},
$$

where $\Omega_{g}$ is some open set around $g \in S E(2)$. Recall that the tangent space at the unity element $e=\left(0,0, e^{i 0}\right)$ is spanned by $T_{e}(G)=\operatorname{span}\left\{\mathbf{e}_{\theta}, \mathbf{e}_{x}, \mathbf{e}_{y}\right\}=\operatorname{span}\{(1,0,0),(0,1,0)$, 
(a) Left-invariance of tangent vectors to curves:

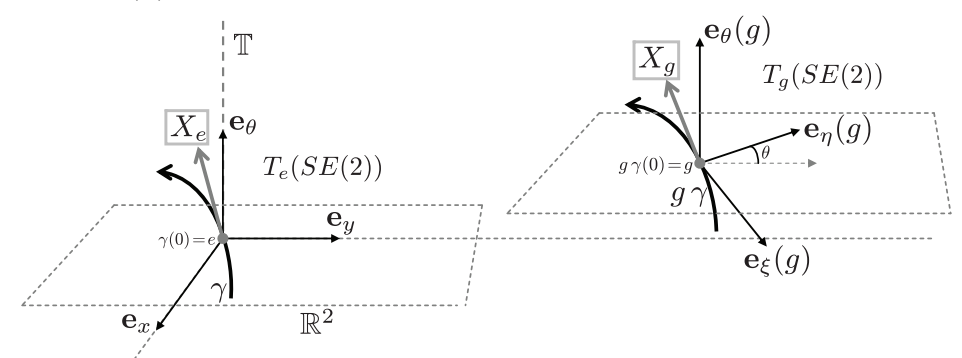

(b) Left-invariance of tangent vectors considered as differential operators:

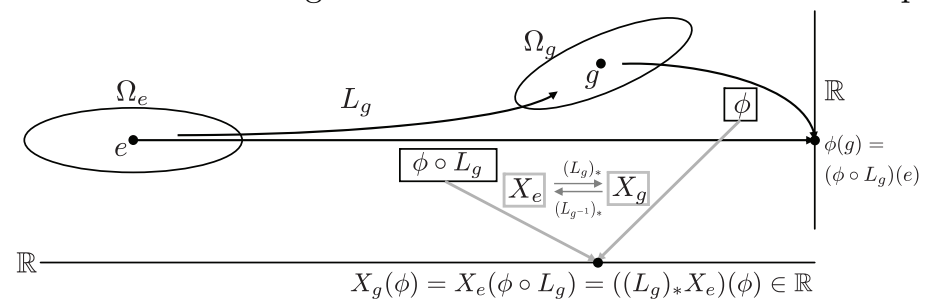

FIG. 4. Left-invariant vector fields on $S E(2)$, where we both consider the tangent vectors tangent to curves, that is, $X_{g}=c^{1} \mathbf{e}_{\theta}(g)+$ $c^{2} \mathbf{e}_{\xi}(g)+c^{3} \mathbf{e}_{\eta}(g)$ for all $g \in S E(2)$, and as differential operators on locally defined smooth functions, that is, $X_{g}=\left.c^{1} \partial_{\theta}\right|_{g}+\left.c^{2} \partial_{\xi}\right|_{g}+$ $\left.c^{3} \partial_{\eta}\right|_{g}$ for all $g \in S E(2)$. We see that the push-forward of the left multiplication connects the tangent space $T_{e}(S E(2))$ to all tangent spaces $T_{g}(S E(2))$.

$(0,0,1)\}$. By the general recipe of constructing left-invariant vector fields from elements in the Lie algebra $T_{e}(G)$ (via the derivative of the right regular representation) we get the following basis for the space $\mathcal{L}(\mathrm{SE}(2))$ of left-invariant vector fields:

$$
\left\{\mathcal{A}_{1}, \mathcal{A}_{2}, \mathcal{A}_{3}\right\}=\left\{\partial_{\theta}, \partial_{\xi}, \partial_{\eta}\right\}=\left\{\partial_{\theta}, \cos \theta \partial_{x}+\sin \theta \partial_{y},-\sin \theta \partial_{x}+\cos \theta \partial_{y}\right\},
$$

with $\xi=x \cos \theta+y \sin \theta, \eta=-x \sin \theta+y \cos \theta$. More precisely, the left-invariant vector fields are given by

$$
\mathbf{e}_{\theta}\left(\mathbf{x}, e^{i \theta}\right)=\mathbf{e}_{\theta}, \quad \mathbf{e}_{\xi}\left(\mathbf{x}, e^{i \theta}\right)=\cos \theta \mathbf{e}_{x}+\sin \theta \mathbf{e}_{y}, \quad \mathbf{e}_{\eta}\left(\mathbf{x}, e^{i \theta}\right)=-\sin \theta \mathbf{e}_{x}+\cos \theta \mathbf{e}_{y},
$$

where we identified $T_{g=\left(\mathbf{x}, e^{i \theta}\right)}\left(\mathbb{R}^{2}, e^{i \theta}\right)$ with $T_{e}\left(\mathbb{R}^{2}, e^{i 0}\right)$ and $T_{g=\left(\mathbf{x}, e^{i \theta}\right)}(\mathbf{x}, \mathbb{T})$ with $T_{e}(\mathbf{0}, \mathbb{T})$, by parallel transport (on $\mathbb{R}^{2}$, respectively $\mathbb{T}$ ). We can always consider these vector fields as differential operators; see Figure 4 . This means that one can always replace $\mathbf{e}_{i}$ by $\partial_{i}$, $i=\theta, \xi, \eta$. Summarizing, we see that for left-invariant vector fields, the tangent vector at $g$ is related to the tangent vector at $e$ by (3.2). Equality (3.2) sets the isomorphism between $T_{e}(S E(2))$ and $\mathcal{L}(S E(2))$, as $A_{i} \leftrightarrow \mathcal{A}_{i}, i=1,2,3$ implies $\left[A_{i}, A_{j}\right] \leftrightarrow\left[\mathcal{A}_{i}, \mathcal{A}_{j}\right]$, $j=1,2,3$; recall (3.1). Moreover it is easily verified that

$$
\left[\mathcal{A}_{1}, \mathcal{A}_{2}\right]=\mathcal{A}_{1} \mathcal{A}_{2}-\mathcal{A}_{2} \mathcal{A}_{1}=\mathcal{A}_{3}, \quad\left[\mathcal{A}_{1}, \mathcal{A}_{3}\right]=-\mathcal{A}_{2}, \quad\left[\mathcal{A}_{2}, \mathcal{A}_{3}\right]=0 .
$$

See Figure 4 for a geometric explanation of left-invariant vector fields, both considered as tangent vectors to curves in $S E(2)$ and as differential operators on locally defined smooth functions. 
Next we follow our general theory for left-invariant scale spaces on Lie groups, see [19], and set the following quadratic form on $\mathcal{L}(S E(2))$ :

$$
Q^{\mathbf{D}, \mathbf{a}}\left(\mathcal{A}_{1}, \mathcal{A}_{2}, \mathcal{A}_{3}\right)=\sum_{i=1}^{3}\left(-a_{i} \mathcal{A}_{i}+\sum_{j=1}^{3} D_{i j} \mathcal{A}_{i} \mathcal{A}_{j}\right), a_{i}, D_{i j} \in \mathbb{R}, D:=\left[D_{i j}\right] \geq 0, D^{T}=D,
$$

and consider the only linear left-invariant second-order evolution equations

$$
\left\{\begin{array}{l}
\partial_{s} W=Q^{\mathbf{D}, \mathbf{a}}\left(\mathcal{A}_{1}, \mathcal{A}_{2}, \mathcal{A}_{3}\right) W \\
W(\cdot, s=0)=\mathcal{W}_{\psi} f(\cdot)
\end{array}\right.
$$

where $W: S E(2) \times \mathbb{R}^{+} \rightarrow \mathbb{C}$, with corresponding resolvent equations (obtained by a Laplace transform over $s$ ):

$$
P=\alpha\left(Q^{\mathbf{D}, \mathbf{a}}\left(\mathcal{A}_{1}, \mathcal{A}_{2}, \mathcal{A}_{3}\right)-\alpha I\right)^{-1} \mathcal{W}_{\psi} f .
$$

These resolvent equations are relevant since for the cases $\mathbf{a}=\mathbf{0}$ they correspond to first-order Tikhonov regularizations on $S E(2)$, 19, 10. They also have an important probabilistic interpretation, as we will explain later on in this section.

By our results in 20], the solutions of these left-invariant evolution equations are given by $S E(2)$-convolution with the corresponding Green's function $K_{s}^{D, \mathbf{a}}$ :

$$
\begin{aligned}
& W(g, s)=\left(K_{s}^{\mathbf{D}, \mathbf{a}} *_{S E(2)} U\right)(g)=\int_{S E(2)} K_{s}^{\mathbf{D}, \mathbf{a}}\left(h^{-1} g\right) U(h) \mathrm{d} \mu_{S E(2)}(h), \\
& =\int_{\mathbb{R}^{2}} \int_{0}^{2 \pi} K_{s}^{\mathbf{D}, \mathbf{a}}\left(R_{\theta^{\prime}}^{-1}\left(\mathbf{x}-\mathbf{x}^{\prime}\right), e^{i\left(\theta-\theta^{\prime}\right)}\right) U\left(\mathbf{x}^{\prime}, e^{i \theta^{\prime}}\right) \mathrm{d} \theta^{\prime} \mathrm{d} \mathbf{x}^{\prime} g=\left(\mathbf{x}, e^{i \theta}\right), \\
& P_{\alpha}(g)=\left(R_{\alpha, \mathbf{D}, \mathbf{a}} *_{S E(2)} U\right)(g), \quad R_{\alpha, \mathbf{D}, \mathbf{a}}=\alpha \int_{0}^{\infty} K_{s}^{\mathbf{D}, \mathbf{a}} e^{-\alpha s} \mathrm{~d} s .
\end{aligned}
$$

Here $\alpha>0$ is the parameter in the Laplace domain, since, at least formally, one has

$$
\begin{aligned}
\int_{0}^{\infty} W(g, s) e^{-\alpha s} \mathrm{~d} s & =\int_{0}^{\infty} e^{s Q^{\mathbf{D}, \mathbf{a}}\left(\mathcal{A}_{1}, \mathcal{A}_{2}, \mathcal{A}_{3}\right)} W(g, 0) e^{-\alpha s} \mathrm{~d} s \\
& =\alpha\left(Q^{\mathbf{D}, \mathbf{a}}\left(\mathcal{A}_{1}, \mathcal{A}_{2}, \mathcal{A}_{3}\right)-\alpha I\right)^{-1} W(g, 0)=P_{\alpha}(g),
\end{aligned}
$$

which puts the connection between (3.8) and (3.7).

In the special case $D_{i j}=\delta_{i 1} \delta_{j 1}, \mathbf{a}=(0,1,0)$, our evolution equation (3.6) is the Kolmogorov equation

$$
\left\{\begin{array}{l}
\partial_{s} W(g, s)=\left(\partial_{\xi}+D_{11} \partial_{\theta}^{2}\right) W(g, s), \quad g \in S E(2), s>0 \\
W(g, 0)=U(g)
\end{array}\right.
$$

of Mumford's direction process, [4],

$$
\left\{\begin{array}{l}
\mathbf{X}(s)=X(s) \mathbf{e}_{x}+Y(s) \mathbf{e}_{y}=\mathbf{X}(0)+\int_{0}^{s} \cos \Theta(\tau) \mathbf{e}_{x}+\sin \Theta(\tau) \mathbf{e}_{y} \mathrm{~d} \tau \\
\Theta(s)=\Theta(0)+\sqrt{s} \sqrt{2 D_{11}} \epsilon_{\theta}, \quad \epsilon_{\theta} \sim \mathcal{N}(0,1),
\end{array}\right.
$$

for contour completion, the explicit solutions of which we have derived in 20.

In this article, however, we are primarily interested in the explicit solutions of the case $D_{i j}=D_{i i} \delta_{i j}, i, j \in\{1,2,3\}, D_{33}=0, \mathbf{a}=\mathbf{0}$ in which case our evolution equation (3.6) 
becomes the forward Kolmogorov equation

$$
\left\{\begin{array}{l}
\partial_{s} W(g, s)=\left(D_{11}\left(\partial_{\theta}\right)^{2}+D_{22}\left(\partial_{\xi}\right)^{2}\right) W(g, s), \\
W(g, 0)=\mathcal{W}_{\psi} f(g)
\end{array}\right.
$$

of the following stochastic process for contour enhancement:

$$
\left\{\begin{array}{l}
\mathbf{X}(s)=\mathbf{X}(0)+\sqrt{2 D_{22}} \epsilon_{\xi} \int_{0}^{s}\left(\cos \Theta(\tau) \mathbf{e}_{x}+\sin \Theta(\tau) \mathbf{e}_{y}\right) \frac{1}{2 \sqrt{\tau}} \mathrm{d} \tau, \\
\Theta(s)=\Theta(0)+\sqrt{s} \sqrt{2 D_{11}} \epsilon_{\theta},
\end{array}\right.
$$

with the standard normal random variables $\epsilon_{\xi} \sim \mathcal{N}(0,1)$ and $\epsilon_{\theta} \sim \mathcal{N}(0,1), D_{11}, D_{22}>0$.

Here we note that contour-completion processes (cf. (3.10) are designed for completion of contours due to occlusion, so one prefers a deterministic drift of the oriented random walker along the preferred positive direction $\cos (\theta) \mathbf{e}_{x}+\sin (\theta) \mathbf{e}_{y}$ in the spatial plane, whereas contour-enhancement processes (cf. (3.12) ) are designed for noise removal by anisotropic diffusion, in which case the stochastic movement of the oriented random walker is bi-directional along the span of $\cos (\theta) \mathbf{e}_{x}+\sin (\theta) \mathbf{e}_{y}$.

In general the evolution equations (3.6) are the forward Kolmogorov equations of all linear left-invariant stochastic processes on $S E(2)$, as explained in [20, 2]. All these cases correspond to continuous stochastic processes such as (3.10) and (3.12).

They can be considered as limiting cases of the following discrete stochastic processes on $S E(2)$ :

$$
\left\{\begin{array}{l}
G_{n+1}:=\left(\mathbf{X}_{n+1}, \Theta_{n+1}\right)=G_{n}+\left.\Delta s \sum_{i=1}^{d} a_{i} \mathbf{e}_{i}\right|_{G_{n}}+\left.\sqrt{\Delta s} \sum_{i=1}^{d} \epsilon_{i, n+1} \sum_{j=1}^{d} \sigma_{j i} \mathbf{e}_{j}\right|_{G_{n}}, \\
G_{0}=\left(\mathbf{X}_{0}, \Theta_{0}\right),
\end{array}\right.
$$

where $n=1, \ldots, N-1, N \in \mathbb{N}$ denotes the number of steps with step-size $\Delta s>0$, $\sigma=\sqrt{2 D}$ is the unique symmetric positive (semi)-definite matrix such that $\sigma^{2}=$ $2 D,\left\{\epsilon_{i, n+1}\right\}_{i=1 \ldots d, n=1, \ldots N-1}$ are independent normally distributed $\epsilon_{i, n+1} \sim \mathcal{N}(0,1)$ and $\left.\mathbf{e}_{1}\right|_{G_{n}}$

$=(0,0,1),\left.\mathbf{e}_{2}\right|_{G_{n}}=\left(\cos \Theta_{n}, \sin \Theta_{n}, 0\right),\left.\mathbf{e}_{3}\right|_{G_{n}}=\left(-\sin \Theta_{n}, \cos \Theta_{n}, 0\right)$.

We wrote the discrete processes in the form (3.13) to stress that the continuous processes (3.12) and (3.10) directly arise by recursion and taking the limit $N \rightarrow \infty$. In more explicit form in $(x, y, \theta)$-coordinates they read:

$$
\begin{aligned}
& \left(\begin{array}{c}
X_{n+1} \\
Y_{n+1} \\
\Theta_{n+1}
\end{array}\right)=\left(\begin{array}{c}
X_{n} \\
Y_{n} \\
\Theta_{n}
\end{array}\right)+\Delta s \mathrm{R}_{\Theta_{n}}\left(\begin{array}{c}
a_{2} \\
a_{3} \\
a_{1}
\end{array}\right)+\sqrt{\Delta s}\left(\mathrm{R}_{\Theta_{n}}\right)^{T} \sigma \mathrm{R}_{\Theta_{\mathrm{n}}}\left(\begin{array}{c}
\epsilon_{2, n+1} \\
\epsilon_{3, n+1} \\
\epsilon_{1, n+1}
\end{array}\right) \\
& \text { with } \mathrm{R}_{\theta}=\left(\begin{array}{ccc}
\cos \theta & -\sin \theta & 0 \\
\sin \theta & \cos \theta & 0 \\
0 & 0 & 1
\end{array}\right) .
\end{aligned}
$$

In this article we shall mainly restrict ourselves to the case $d=2$ (or equivalently $d=3$ and $D_{i 3}=0, i=1,2,3$ and $\left.a_{3}=0\right)$ so that the trajectories only use the horizontal part, spanned by $\left\{\left.\mathbf{e}_{1}\right|_{g},\left.\mathbf{e}_{2}\right|_{g}\right\} \equiv\left\{\mathbf{e}_{\theta}(g), \mathbf{e}_{\xi}(g)\right\}$, of each tangent space $\left.T_{g}(S E(2)), g \in S E(2)\right)$. Occasionally, we shall also consider $d=3, a_{3}=0$ and $D_{33}>0$. See Figure 5.

With respect to this connection to probability theory we note that $W(g, s)$ represents the probability density of finding an oriented random walker 5 (traveling with unit speed,

\footnotetext{
${ }^{5}$ That is, a random walker in the space $S E(2)$ that is only allowed to move along horizontal curves, i.e., along curves with tangent vectors within $\operatorname{span}\left\{\partial_{\theta}, \partial_{\xi}\right\}$, which is the horizontal subspace if we apply
} 

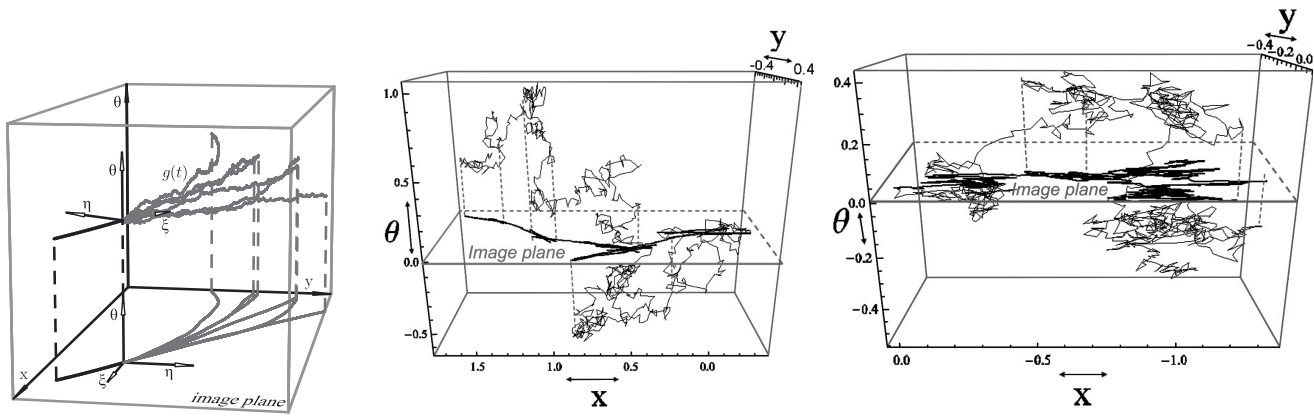

FIG. 5. Left: six random walks in $S E(2)=\mathbb{R}^{2} \rtimes \mathbb{T}$ (and their projection on $\mathbb{R}^{2}$ ) of direction processes for contour completion by Mumford [4] with $\mathbf{a}=\left(\kappa_{0}, 1,0\right), D=\operatorname{diag}\left\{D_{11}, D_{22}, D_{33}\right\}$ for various parameter settings of $\kappa_{0} \geq 0$ and $D_{i i}>0$. Middle: one random walk $(N=500$ steps, with step-size $\Delta s=0.005)$ and its projection to the image plane of the linear left-invariant stochastic process for contour enhancement within $S E(2)$ with parameter settings $D_{11}=D_{22}=\frac{1}{2}$ and $D_{33}=0$ (corresponding to the cortical model of Citti and Sarti for contour enhancement, [10]). Right: one random walk $(N=800$ steps, with step-size $\Delta s=0.005)$ of the stochastic process with parameter settings $D_{11}=\frac{1}{2} \sigma_{\theta}^{2}, D_{22}=\frac{1}{2} \sigma_{\xi}^{2}$, $D_{33}=\frac{1}{2} \sigma_{\eta}^{2}$, with $\sigma_{\theta}=0.75, \sigma_{\xi}=1, \sigma_{\eta}=0.5$ (other parameters have been set to zero). Appropriate averaging of infinitely many of these sample paths yields the Green's functions; see Figure 7 of the forward Kolmogorov equations (3.6). Note that Mumford's direction process is the only linear left-invariant stochastic process on $\mathrm{SE}(2)$ whose sample path projections on the image plane are differentiable. For contour completion this is desirable; see 2. However, the Green's function of all linear left-invariant processes (so also the ones for contour enhancement) are infinitely differentiable on $S E(2) \backslash\{e\}$ iff the Hörmander condition as we will discuss in Section 5.3 see (5.19), is satisfied.

which allows us to identify traveling time with arc-length $s$ ) at position $g$ and traveling time $s>0$ given the initial distribution $W(\cdot, 0)=\mathcal{W}_{\psi} f$, whereas $P(g)$ represents the unconditional probability density of finding an oriented random walker at position $g$ given the initial distribution $W(\cdot, 0)=\mathcal{W}_{\psi} f$ regardless of its traveling time. To this end we note that traveling time $T$ in a Markov process is negatively exponentially distributed:

$$
P(T=s)=\alpha e^{-\alpha s},
$$

since this is the only continuous memoryless distribution. A simple calculation yields:

$$
\begin{aligned}
& P(x, y, \theta \mid U \text { and } T=s)=\left(K_{s}^{D_{11}} *_{S E(2)} U\right)(x, y, \theta), \\
& P(x, y, \theta \mid U)=\int_{0}^{\infty} P(x, y, \theta \mid U \text { and } T=s) P(T=s) \mathrm{d} s=\left(R_{\alpha, D_{11}} *_{S E(2)} U\right)(x, y, \theta) \\
& \text { with } R_{\alpha, D_{11}}=\alpha \int_{\mathbb{R}^{+}} K_{s}^{D_{11}} e^{-\alpha s} \mathrm{~d} s .
\end{aligned}
$$

the Cartan connection on $P_{Y}=(S E(2), S E(2) / Y, \pi, R)$; see part II, 22]. In previous work in the field of image processing, 15, 16, we called these random walkers "oriented grey-value particles". 
For exact solutions for the resolvent equations (3.7) (in the case of Mumford's direction process), approximations, and fast numerical algorithms (related to Fourier-transform on $S E(2)$ ), see [20]. For more details on efficient computation schemes of $S E(2)$-convolutions in general and Fourier transforms on $S E(2)$, we refer to 9 .

Finally, we recall from 20, that both diffusion and convection in the evolutions (3.6) take place along the exponential curves in $S E(2)$. These well-known curves are circular spirals and straight lines; for explicit formulas of the exponential curves, see [20, eq. 3.7].

4. Image enhancement via left-invariant evolution equations on invertible orientation scores. In Section 2 we have constructed a stable transformation between images $f$ and corresponding orientation scores $\mathcal{W}_{\psi} f$. This enables us to relate operators $\Upsilon$ on images to operators $\Phi$ on orientation scores in a robust manner; see Figure 6. Let $\mathcal{B}\left(\mathbb{L}_{2}(S E(2))\right)$ denote the space of all bounded linear operators on $\mathbb{L}_{2}(S E(2))$.

It is easily verified that $\mathcal{W}_{\psi} \circ \mathcal{U}_{g}=\mathcal{L}_{g} \circ \mathcal{W}_{\psi}$ for all $g \in S E(2)$, where the left-regular representation $\mathcal{L}: G \rightarrow \mathcal{B}\left(\mathbb{L}_{2}(S E(2))\right)$ is given by $\mathcal{L}_{g} U(h)=U\left(g^{-1} h\right)$. Consequently, the effective operator on the image $\Upsilon$ is Euclidean invariant if and only if the operator on the orientation score is left-invariant, i.e.,

$$
\Upsilon \circ \mathcal{U}_{g}=\mathcal{U}_{g} \circ \Upsilon \text { for all } g \in S E(2) \Leftrightarrow \Phi \circ \mathcal{L}_{g}=\mathcal{L}_{g} \circ \Phi \text { for all } g \in S E(2) ;
$$

see [14, Thm. 21, p.153].

The diffusions discussed in the previous section, Section 3, can be used to construct a suitable operator $\Phi$ on the orientation scores. At first glance the linear diffusions themselves (with a certain stopping time $t>0$ ) and their resolvents (with parameter $\alpha>0$ ) seem to be suitable candidates for operators on orientation scores, as they follow from stochastic processes for contour enhancement. However, if the operator $\Phi$ is leftinvariant (which is required, see Figure 6) and linear, then the effective operator $\Upsilon$ is translation and rotation invariant, boiling down to an isotropic convolution on the original image. Clearly, in such a case one does not need any orientation scores.

So our operator $\Phi$ must be left-invariant and nonlinear and still we would like to relate such an operator to stochastic processes on $S E(2)$ as we discussed in the previous section. Therefore we consider the operators (with $\underline{\mathcal{A}}:=\left\{\mathcal{A}_{1}, \mathcal{A}_{2}, \mathcal{A}_{3}\right\}$ )

$$
\begin{aligned}
& \Phi(U, V)=\left(Q^{D, \mathbf{a}}(\underline{\mathcal{A}})-\alpha I\right)^{-1}(\chi(U))\left(\left(Q^{D, \mathbf{a}}(\underline{\mathcal{A}})\right)^{*}-\alpha I\right)^{-1}(\chi(V)), \\
& =\left(R_{\alpha, D,-\mathbf{a}} *_{S E(2)}(\chi(U))\right) \cdot\left(R_{\alpha, D,-\mathbf{a} *_{S E(2)}}(\chi(V))\right),
\end{aligned}
$$

where $U$ (the source distribution) and $V$ (the sink distribution) denote two initial distributions on $S E(2)$ and where $\chi$ is a monotonic, homogeneou 6 grey-value transformation on orientation scores such as $\chi(U)(x, y, \theta)=F(U(x, y, \theta))$, with $F: \mathbb{R} \rightarrow \mathbb{R}$ given by $F(I)=|I|^{p} \operatorname{sign}(I), I \in \mathbb{R}$, for some $p>1$. The function $\Phi(U, V) \in \mathbb{L}_{2}(S E(2))$ can be considered as the "collision distribution" obtained from collision of the forwardly evolving source distribution $U$ and backwardly evolving sink distribution $V$, similar to [7. Originally, this idea was first developed for contour-completion processes in [57]. To motivate the word "collision distribution" we recall from (3.16) that $\left(Q^{D, \mathbf{a}}(\underline{\mathcal{A}})-\alpha I\right)^{-1}(\chi(U))(g)$ represents the unconditional probability density of finding a random walker (regardless

\footnotetext{
${ }^{6}$ To ensure grey-scaling $f \mapsto \lambda f, \lambda>0$ covariance of the effective operator $\Upsilon_{\psi}$ (4.3).
} 


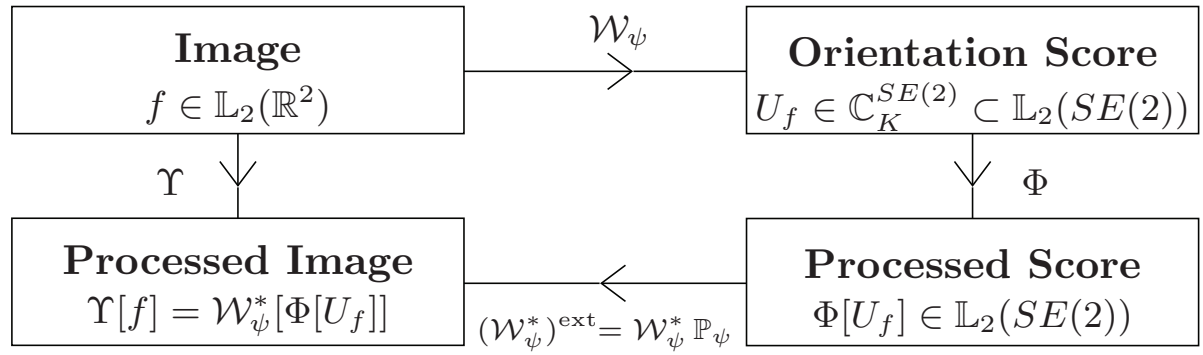

Contour enhancement input image $f$

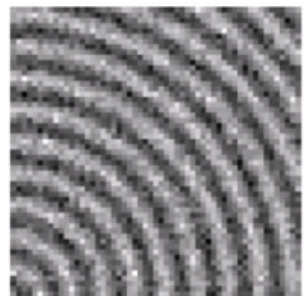

output image $\Upsilon f=\mathcal{W}_{\phi}^{*} \Phi \mathcal{W}_{\phi} f$

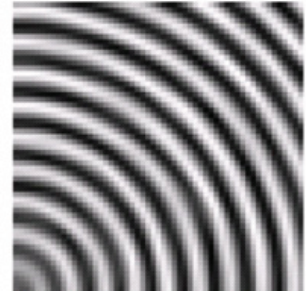

Contour completion input image $f \quad$ output image $\Upsilon f=\mathcal{W}_{\phi}^{*} \Phi \mathcal{W}_{\phi} f$
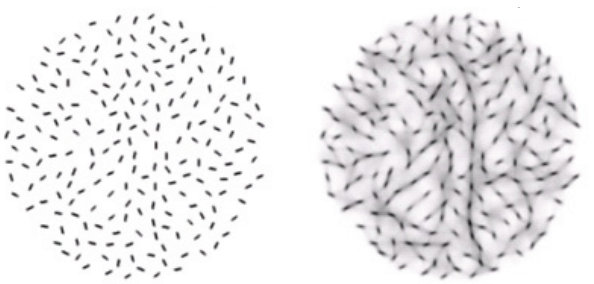

Fig. 6. Top Row: The complete scheme; for admissible anisotropic wavelets $\psi$ the linear map $\mathcal{W}_{\psi}$ is unitary from $\mathbb{L}_{2}\left(\mathbb{R}^{2}\right)$ onto a closed subspace $\mathbb{C}_{K}^{S E(2)}$ of $\mathbb{L}_{2}(S E(2))$. So we can uniquely relate a transformation $\Phi: \mathbb{C}_{K}^{S E(2)} \rightarrow \mathbb{C}_{K}^{S E(2)}$ on an orientation score to a transformation on an image $\Upsilon_{\psi}=\left(\mathcal{W}_{\psi}^{*}\right)^{\text {ext }} \circ \Phi \circ \mathcal{W}_{\psi}$, where $\left(\mathcal{W}_{\psi}^{*}\right)^{\text {ext }}$ is given by (4.4). Here we take $\Phi$ as a concatenation of nonlinear invertible grey-value transforms and linear left-invariant evolutions (4.3) with $U=V=\Re\left\{\mathcal{W}_{\psi}(f)\right.$. Bottom row: Automated contour enhancement (left) and completion (right). Part II of this article offers a more adaptive alternative to the operator $\Phi$ defined by (4.3); one can set the operator $\Phi$ as the adaptive evolution operator $\mathcal{W}_{\psi} f \mapsto u\left(x, y, e^{i \theta}, t\right)$ defined by the nonlinear adaptive left-invariant evolution equation with certain stopping time $t>0$.

of its traveling time) at position $g$ starting from the initial distribution $\chi(U)$. Now the probability density of finding both a random walker evolving independently from a source distribution $\chi(U)$ and a random walker from the sink distribution $\chi(V)$ (regardless of its traveling time) is up to normalization equivalent to the direct product of the probability densities. For a clear connection between the well-known Brownian-bridge measures in probability theory on manifolds [58, and the measures induced by collision distributions, both defined on the manifold $S E(2)$, see [21, App. B, pp. 67-69].

In contrast to earlier work [21, [20, 6], we shall restrict ourselves here to the case where both source and sink equal the real-valued part of the orientation score of the original image $f$, i.e. $U=V=\Re\left\{\mathcal{W}_{\psi} f\right\}$, so that the effective operator on the image $f \in \mathbb{L}_{2}\left(\mathbb{R}^{2}\right)$ becomes

$$
\Upsilon_{\psi}(f)=\mathcal{W}_{\psi}^{*}\left[\left(\left(Q^{D, \mathbf{a}}(\underline{A})-\alpha I\right)^{-1}\left(\chi\left(\Re\left\{\mathcal{W}_{\psi} f\right\}\right)\right)\right) \cdot\left(\left(Q^{D, \mathbf{a}}(\underline{A})\right)^{*}-\alpha I\right)^{-1}\left(\chi\left(\Re\left\{\mathcal{W}_{\psi} f\right\}\right)\right)\right] .
$$


Here we note that the imaginary part, which we discard by setting $U=V=\Re\left\{\mathcal{W}_{\psi} f\right\}$, of the orientation score does not play a role in the reconstruction.

In part II of this article, 22, we shall consider more sophisticated alternatives to the operator given by (4.3). In this part, however, we restrict ourselves to the case (4.3), since this is much easier to analyse and also easier to implement as it requires two group convolutions (recall (3.8)) with the corresponding Green's functions, which we shall explicitly derive in the next section.

The relation between image and orientation score remains bijective if we ensure that the operator on the orientation score again provides an orientation score of an image: Let $\mathbb{C}_{K}^{S E(2)}$ denote the space of orientation scores within $\mathbb{L}_{2}(S E(2))$. Recall from Section 2 that we use this notation since the space of orientation scores generated by a proper wavelet $\psi$ is the unique reproducing kernel space on $S E(2)$ with reproducing kernel, 14, pp. 221-222, pp. 120-122], [12,

$$
K(g, h)=\left(\mathcal{U}_{g} \psi, \mathcal{U}_{h} \psi\right) .
$$

An operator on an orientation score is 1-to-1 related to an operator on an image iff $\Phi$ maps $\mathbb{C}_{K}^{S E(2)}$ into $\mathbb{C}_{K}^{S E(2)}$. However, in general it is very hard to directly obtain operators that leave the space of orientation scores $\mathbb{C}_{K}^{S E(2)}$ invariant. Therefore we naturally extend the reconstruction to $\mathbb{L}_{2}(S E(2))$ :

$$
\left(\mathcal{W}_{\psi}^{*}\right)^{e x t} U(g)=\mathcal{F}^{-1}\left[\boldsymbol{\omega} \mapsto \int_{0}^{2 \pi} \mathcal{F}\left[U\left(\cdot, e^{i \theta}\right)\right](\boldsymbol{\omega}) \mathcal{F}\left[\mathcal{R}_{e^{i \theta}} \psi\right](\boldsymbol{\omega}) \mathrm{d} \theta M_{\psi}^{-1}(\boldsymbol{\omega})\right],
$$

for all $U \in \mathbb{L}_{2}(S E(2))$. Consequently, the effective part of an operator $\Phi: \mathbb{C}_{K}^{S E(2)} \rightarrow$ $\mathbb{L}_{2}(S E(2))$ on an orientation score is in fact $\mathbb{P}_{\psi} \Phi: \mathbb{C}_{K}^{S E(2)} \rightarrow \mathbb{C}_{K}^{S E(2)}$, where $\mathbb{P}_{\psi}=$ $\mathcal{W}_{\psi}\left(\mathcal{W}_{\psi}^{*}\right)^{\text {ext }}$ is the orthogonal projection of $\mathbb{L}_{2}(S E(2))$ onto $\mathbb{C}_{K}^{S E(2)}$. Here we note that the range $\mathcal{R}\left(\mathcal{W}_{\psi}\right)$ of $\mathcal{W}_{\psi}$ equals $\mathcal{R}\left(\mathcal{W}_{\psi}\right)=\mathbb{C}_{K}^{S E(2)}=\mathcal{R}\left(\mathbb{P}_{\psi}\right)=\mathcal{N}\left(I-\mathbb{P}_{\psi}\right)$ and thereby (since the null space of a bounded operator is always closed) the space of orientation scores $\mathbb{C}_{K}^{S E(2)}$ is a closed subspace of $\mathbb{L}_{2}(S E(2))$. Furthermore, we note that $\left(\mathbb{P}_{\psi} \Phi\right)(g)=$ $\int_{S E(2)} K(g, h) \Phi(h) \mathrm{d} h$.

Now recall that $\Phi$ must be left-invariant because of (4.1). It is not difficult to show that the only linear left-invariant kernel operators on $\mathbb{L}_{2}(S E(2))$ are $S E(2)$-convolutions, which are given by (3.8). Even these $S E(2)$-convolutions do not leave the space of orientation scores $\mathbb{C}_{K}^{S E(2)}$ invariant. Although for all $f \in \mathbb{L}_{2}\left(\mathbb{R}^{2}\right), g \in S E(2), k \in$ $\mathbb{L}_{1}(S E(2))$, one has

$$
\begin{aligned}
\left(k *_{S E(2)} \mathcal{W}_{\psi} f\right)(g) & =\int_{S E(2)}\left(\mathcal{U}_{h} \psi, f\right)_{\mathbb{L}_{2}\left(\mathbb{R}^{2}\right)} k\left(h^{-1} g\right) \mathrm{d} \mu_{S E(2)}(h) \\
& =\left(\int_{S E(2)} \mathcal{U}_{g \tilde{h}-1} \psi k(\tilde{h}) \mathrm{d} \mu_{S E(2)}(\tilde{h}), f\right)_{\mathbb{L}_{2}\left(\mathbb{R}^{2}\right)} \\
& =\left(\mathcal{U}_{g} \tilde{\psi}, f\right)_{\mathbb{L}_{2}\left(\mathbb{R}^{2}\right)}=\mathcal{W}_{\tilde{\psi}} f(g),
\end{aligned}
$$

where $\tilde{\psi}=\int_{S E(2)} \mathcal{U}_{\tilde{h}^{-1}} \psi k(\tilde{h}) \mathrm{d} \mu_{S E(2)}(\tilde{h})$, the reproducing kernel space associated to $\tilde{\psi}$ will in general not coincide with the reproducing kernel space associated to $\psi$. 
5. The heat kernels on $S E(2)$. In Section 5.1 we will present the exact formulas of the Green's functions and their resolvents for linear anisotropic diffusion on the group $S E(2)$, which do not seem to appear in the literature. Although the exact resolvent diffusion kernels, which take care of Tikhonov regularization on $\mathrm{SE}(2)$, cf. 19, are expressed in only four Mathieu functions, we also derive the corresponding Heisenberg approximative resolvent diffusion kernels, in Section 5.2. These approximate Green's functions are Green's functions on the space of positions and velocities rather than Green's functions on the space of positions and orientations and arise by replacing $\cos \theta$ by 1 and $\sin \theta$ by $\theta$ in the generators. In the context of contour completion this was first proposed in [53] and here we will mainly focus on the contour-enhancement case. Although these approximative Green's functions are not as simple as in the contour-completion case, [20, ch:4.3], they are more suitable if it comes to fast implementations. For comparison between the exact resolvent heat kernels and their approximations, see Figure 7 .

5.1. The exact heat kernels on $S E(2)=\mathbb{R}^{2} \rtimes S O(2)$. In this section we will derive the heat kernels $K_{s}^{D}: S E(2) \rightarrow \mathbb{R}^{+}$and the corresponding resolvent kernels $R_{\alpha, D}$ : $S E(2) \rightarrow \mathbb{R}^{+}$on $S E(2)$. Recall that $S E(2)$-convolution with these kernels, see (3.8), provides the solutions of the forward Kolmogorov equations (3.11) and recall that $R_{\alpha, D}=$ $\alpha \int_{0}^{\infty} K_{s}^{D} e^{-\alpha s} \mathrm{~d} s$. In this chapter we set $D$ as a constant diagonal matrix. Although $D_{33}=0$ (as in Section 3, (3.11) ) has our main interest we also consider the case $D_{33} \geq 0$.

The kernels $K_{s}^{D}$ and $R_{\alpha, D}$ are the unique solutions of the following problems:

$$
\begin{aligned}
& \left\{\begin{array}{l}
\left(-D_{11}\left(\partial_{\theta}\right)^{2}-D_{22}\left(\partial_{\xi}\right)^{2}-D_{33}\left(\partial_{\eta}\right)^{2}+\alpha\right) R_{\alpha, D}=\alpha \delta_{e} \\
R_{\alpha, D}(\cdot, \cdot, 0)=R_{\alpha, D}(\cdot, \cdot, 2 \pi) R_{\alpha, D} \in \mathbb{L}_{1}(S E(2)),
\end{array}\right. \\
& \left\{\begin{array}{l}
\partial_{s} K_{s}^{D}=\left(D_{11}\left(\partial_{\theta}\right)^{2}+D_{22}\left(\partial_{\xi}\right)^{2}+D_{33}\left(\partial_{\eta}\right)^{2}\right) K_{s}^{D} \\
\lim _{s \downarrow 0} K_{s}^{D}=\delta_{e} \\
K_{s}^{D} \in \mathbb{L}_{1}(S E(2)) .
\end{array}\right.
\end{aligned}
$$

The first step here is to perform a Fourier transform with respect to the spatial part $\equiv \mathbb{R}^{2}$ of $S E(2)=\mathbb{R}^{2} \rtimes \mathbb{T}$, so that we obtain $\hat{R}_{\alpha, D}, \hat{K}_{s}^{D} \in \mathbb{L}_{2}(S E(2)) \cap C(S E(2))$ :

$$
\begin{aligned}
& \hat{K}_{s}^{D}\left(\omega_{1}, \omega_{2}, \theta\right)=\mathcal{F}\left[K_{s}^{D}(\cdot, \cdot, \theta)\right]\left(\omega_{1}, \omega_{2}\right), \\
& \hat{R}_{\alpha, D}\left(\omega_{1}, \omega_{2}, \theta\right)=\mathcal{F}\left[R_{\alpha, D}(\cdot, \cdot, \theta)\right]\left(\omega_{1}, \omega_{2}\right) .
\end{aligned}
$$

Then $\hat{R}_{\alpha, D}$ and $\hat{K}_{s}^{D}$ satisfy

$$
(\alpha I-\mathcal{B} \boldsymbol{\omega}) \hat{R}_{\alpha, D}=\frac{\alpha}{2 \pi} \delta_{0} \quad \text { and } \partial_{s} \hat{K}_{s}^{D}=\mathcal{B} \boldsymbol{\omega} \hat{K}_{s}^{D}, \lim _{s \downarrow 0} \hat{K}_{s}^{D}(\boldsymbol{\omega}, \theta)=\delta_{e},
$$

where we define the operator

$$
\mathcal{B}_{\boldsymbol{\omega}}=-D_{22} \rho^{2} \cos ^{2}(\varphi-\theta)-D_{33} \rho^{2} \sin ^{2}(\varphi-\theta)+D_{11}\left(\partial_{\theta}\right)^{2}
$$

where we expressed $\boldsymbol{\omega} \in \mathbb{R}^{2}$ in polar coordinates

$$
\boldsymbol{\omega}=(\rho \cos \varphi, \rho \sin \varphi) \in \mathbb{R}^{2},
$$

and where we note that $\mathcal{F}\left(\delta_{e}\right)=\frac{1}{2 \pi} 1_{\mathbb{R}^{2}} \otimes \delta_{0}^{\theta}$. We can rewrite operator $\mathcal{B} \boldsymbol{\omega}$ to a Mathieu operator (corresponding to the well-known Mathieu equation (5.4), 45], 1])

$$
\mathcal{B}_{\boldsymbol{\omega}}=D_{11}\left(\left(\partial_{\theta}\right)^{2}+a I-2 q \cos (2(\varphi-\theta))\right),
$$


where $a=-\frac{\alpha+\left(\rho^{2} / 2\right)\left(D_{22}+D_{33}\right)}{D_{11}}$ and $q=\rho^{2}\left(\frac{D_{22}-D_{33}}{4 D_{11}}\right) \in \mathbb{R}$. Clearly, this unbounded operator (with domain $\mathcal{D}(\mathcal{B} \boldsymbol{\omega})=\mathbb{H}^{2}(\mathbb{T})$ ) is for each fixed $\boldsymbol{\omega} \in \mathbb{R}^{2}$ a symmetric operator of Sturm-Liouville type on $\mathbb{L}_{2}(\mathbb{T})$ :

$$
\left(\mathcal{B}_{\boldsymbol{\omega}}\right)^{*}=\mathcal{B}_{\boldsymbol{\omega}}
$$

Its right inverse extends to a compact selfadjoint operator on $\mathbb{L}_{2}(\mathbb{T})$ and thereby $B \boldsymbol{\omega}$ has the following complete orthogonal basis of eigenfunctions:

$$
\begin{aligned}
& \Theta_{n}^{\boldsymbol{\omega}}(\theta)=\operatorname{me}_{n}(\varphi-\theta, q), \quad n \in \mathbb{Z}, q=\rho^{2}\left(\frac{D_{22}-D_{33}}{4 D_{11}}\right) \in \mathbb{R}, \\
& \mathcal{B}_{\boldsymbol{\omega}} \Theta_{n}^{\boldsymbol{\omega}}=\lambda_{n}^{o} \Theta_{n}^{\boldsymbol{\omega}}, \\
& \lambda_{n}^{\rho}=-a_{n}(q) D_{11}-\frac{\rho^{2}}{2}\left(D_{22}+D_{33}\right) \leq-n^{2} D_{11} \leq 0,
\end{aligned}
$$

where $\operatorname{me}_{n}(z, q)=\mathrm{ce}_{n}(z, q)+i \mathrm{se}_{n}(z, q)$ denotes the well-known Mathieu function (with discrete Floquet exponent $\nu=n$ ), 45, 1, and characteristic values $a_{n}(q)$ which are countable solutions of the corresponding characteristic equations [1, p.723], 45, containing continued fractions. Note that at $\boldsymbol{\omega}=\mathbf{0}$, i.e. $\rho=0$, we have $\operatorname{me}_{n}(z, 0)=e^{i n z}, \lambda_{n}^{0}=n^{2}$.

The functions $q \mapsto a_{n}(q)$ are analytic on the real line. In contrast with the eigenfunction decomposition of the generator of the forward Kolmogorov equation (3.9) of Mumford's direction process [20], we have $q \in \mathbb{R}$ rather than $q \in i \mathbb{R}$ and therefore we will not meet any cumbersome branching points of $a_{n}$. For Taylor expansions of $a_{n}(q)$, see [1, p.730]. For each fixed $\boldsymbol{\omega} \in \mathbb{R}^{2}$ the set $\left\{\Theta_{n}^{\boldsymbol{\omega}}\right\}_{n \in \mathbb{Z}}$ is a complete orthogonal basis for $\mathbb{L}_{2}(\mathbb{T})$ and

$$
\left\langle\delta_{0}, \phi\right\rangle=\phi(0)=\frac{1}{2 \pi} \sum_{n=-\infty}^{\infty}\left(\Theta_{n}^{\boldsymbol{\omega}}, \phi\right) \Theta_{n}^{\boldsymbol{\omega}}(0)
$$

for all test functions $\phi \in \mathcal{D}(\mathbb{T})$. Consequently, the unique solutions of (5.1) are given by

$$
\hat{K}_{s}(\boldsymbol{\omega}, \theta)=\frac{1}{2 \pi} \sum_{n=\infty}^{\infty} \Theta_{n}^{\boldsymbol{\omega}}(\theta) \Theta_{n}^{\boldsymbol{\omega}}(0) e^{\lambda_{n}^{o} s}, \quad \hat{R}_{\alpha, D}(\boldsymbol{\omega}, \theta)=\frac{\alpha}{2 \pi} \sum_{n=-\infty}^{\infty} \frac{\Theta_{n}^{\boldsymbol{\omega}}(\theta) \Theta_{n}^{\boldsymbol{\omega}}(0)}{\alpha-\lambda_{n}^{p}} .
$$

This proves the following result:

Theorem 5.1. Let $D_{11}, D_{22}, D_{33}>0$. Then the heat kernels $K_{s}^{D_{11}, D_{22}, D_{33}}$ on the Euclidean motion group which satisfy

$$
\left\{\begin{array}{l}
\partial_{s} K_{s}^{D_{11}, D_{22}, D_{33}}=\left(D_{11}\left(\partial_{\theta}\right)^{2}+D_{22}\left(\partial_{\xi}\right)^{2}+D_{33}\left(\partial_{\eta}\right)^{2}\right) K_{s}^{D_{11}, D_{22}, D_{33}} \\
K_{s}^{D_{11}, D_{22}, D_{33}}(\cdot, \cdot, 0)=K_{s}^{D_{11}, D_{22}, D_{33}}(\cdot, \cdot, 2 \pi) \text { for all } s>0 \\
\lim _{s \downarrow 0} K_{s}^{D_{11}, D_{22}, D_{33}}(\cdot, \cdot, \cdot)=\delta_{e} \\
K_{s}^{D_{11}, D_{22}, D_{33}} \in \mathbb{L}_{1}(S E(2)), \text { for all } s>0
\end{array}\right.
$$

are given by

$$
K_{s}^{D_{11}, D_{22}, D_{33}}\left(x, y, e^{i \theta}\right)=\mathcal{F}^{-1}\left[\boldsymbol{\omega} \mapsto \hat{K}_{s}^{D_{11}, D_{22}, D_{33}}\left(\boldsymbol{\omega}, e^{i \theta}\right)\right](x, y),
$$

where

$$
\hat{K}_{s}^{D_{11}, D_{22}, D_{33}}\left(\boldsymbol{\omega}, e^{i \theta}\right)=e^{-s(1 / 2)\left(D_{22}+D_{33}\right) \rho^{2}}\left(\sum_{n=-\infty}^{\infty} \frac{\operatorname{me}_{n}(\varphi, q) \mathrm{me}_{n}(\varphi-\theta, q)}{2 \pi} e^{-s a_{n}(q) D_{11}}\right)
$$


with $q=\frac{\rho^{2}\left(D_{22}-D_{33}\right)}{4 D_{11}}$ and $a_{n}(q)$ the Mathieu characteristic with Floquet exponent $n$ and with the property that $K_{s}^{D_{11}, D_{22}, D_{33}}>0$ and

$$
\begin{aligned}
\left\|K_{s}^{D_{11}, D_{22}, D_{33}}\right\|_{\mathbb{L}_{1}(S E(2))} & =\int_{0}^{2 \pi} \hat{K}_{s}^{D_{11}, D_{22}, D_{33}}\left(\mathbf{0}, e^{i \theta}\right) \mathrm{d} \theta \\
& =\sum_{n=-\infty}^{\infty}(2 \pi)^{-1} \int_{0}^{2 \pi} e^{i n \theta} \mathrm{d} \theta e^{-s n^{2} D_{11}}=1 .
\end{aligned}
$$

Consider the case where $D_{11} \downarrow 0$. Then $a_{n}(q) \sim-2 q$ as $q \rightarrow \infty$ and we have

$$
\begin{aligned}
\lim _{D_{11} \downarrow 0} \hat{K}_{s}^{D_{11}, D_{22}, D_{33}}\left(\boldsymbol{\omega}, e^{i \theta}\right) & =e^{-\frac{s}{2}\left(D_{22}+D_{33}\right)\left(\omega_{x}^{2}+\omega_{y}^{2}\right)} e^{-\frac{s}{2}\left(D_{22}-D_{33}\right)\left(\omega_{x}^{2}-\omega_{y}^{2}\right)} \delta_{0}^{\theta} \\
& =e^{-s\left(D_{22} \omega_{x}^{2}+D_{33} \omega_{y}^{2}\right)} \delta_{0}^{\theta}=\hat{K}_{s}^{D_{11}, D_{22}, D_{33}}\left(\boldsymbol{\omega}, e^{i \theta}\right) \delta_{0}^{\theta}
\end{aligned}
$$

Finally, the case $D_{11}=0$ yields the following operation on $\mathbb{L}_{2}(S E(2))$ :

$$
\begin{aligned}
\left(K_{s}^{0, D_{22}, D_{33}} *_{S E(2)} U\right)(g) & =\int_{\mathbb{R}^{2}} G_{t}^{D_{22}, D_{33}}\left(R_{\theta}^{-1}\left(\mathbf{x}-\mathbf{x}^{\prime}\right)\right) U\left(\mathbf{x}^{\prime}, e^{i \theta}\right) \mathrm{d} \mathbf{x}^{\prime} \\
& =\left(\mathcal{R}_{e^{i \theta}} G_{t}^{D_{22}, D_{33}} *_{\mathbb{R}^{2}} f\right)(\mathbf{x})
\end{aligned}
$$

$g=\left(\mathbf{x}, e^{i \theta}\right) \in S E(2)$, where $G_{t}^{D_{22}, D_{33}}(x, y)=G_{t D_{22}}^{d=1}(x) G_{t}^{d=1}(y)$ equals the well-known anisotropic Gaussian kernel or heat kernel on $\mathbb{R}^{n}$, and where $\mathcal{R}_{e^{i \theta}} \phi(\mathbf{x})=\phi\left(R_{\theta}^{-1} \mathbf{x}\right)$ is the left regular action of $S O(2)$ in $\mathbb{L}_{2}\left(\mathbb{R}^{2}\right)$, which corresponds to anisotropic diffusion in each fixed orientation layer $U(\cdot, \cdot, \theta)$ where the axes of anisotropy coincide with the $\xi$ and $\eta$ axes. This operation is, for example, used in image analysis in the framework of channel smoothing [24], 15]. However, also the diffusion kernels with $D_{11}>0$ are interesting for various image processing frameworks such as tensor voting, channel representations and invertible orientation scores, as they allow different orientation layers $\{U(\cdot, \cdot, \theta)\}_{\theta \in[0,2 \pi)}$ to interact. For an illustration of the corresponding resolvent kernel $R_{\alpha, D}$ (with comparison to the approximations in Section [5.2), see Figure 7.

Although expression (5.2) for the exact resolvent kernel $R_{\alpha, D}$ can be related to numerical schemes [20, ch: 5], it is a Fourier series which converges (point-wise) rather slowly in the neighborhood of the unity element. Therefore we shall derive a more suitable series expression than (5.2) for the resolvent kernel $R_{\alpha, D}$ with rapidly decreasing terms. To this end we will unwrap the torus to $\mathbb{R}$ and replace the periodic boundary condition in $\theta$ by an absorbing boundary condition at infinity. Afterwards we shall construct the true periodic solution by explicitly computing (using Floquet's theorem) the series consisting of (rapidly decreasing) $2 \pi$-shifts of the solution with absorbing condition at infinity.

In our explicit formulas for the resolvent kernel $R_{\alpha, D}$ we shall make use of the nonperiodic complex-valued Mathieu function which is a solution of the Mathieu equation

$$
y^{\prime \prime}(z)+[(a-2 q) \cos (2 z)] y(z)=0, \quad a, q \in \mathbb{R}
$$


and which is by definition 7 [45, p.115], [1, p.732], given by

$$
\operatorname{me}_{ \pm \nu}(z, q)=\mathrm{ce}_{\nu}(z, q) \pm i \mathrm{se}_{\nu}(z, q) .
$$

Here $\nu=\nu(a, q)$ equals the Floquet exponent (due to the Floquet Theorem [45, p.101]) of the solution, which means that

$$
\mathrm{me}_{ \pm \nu}(z+\pi, q)=e^{i \nu z} \mathrm{me}_{ \pm \nu}(z, q)
$$

for all $z, q \in \mathbb{R}$.

Theorem 5.2. Let $\alpha>0, D_{22} \geq D_{33}>0, D_{11}>0$. The solution $R_{\alpha, D}^{\infty}: \mathbb{R}^{3} \backslash\{0,0,0\} \rightarrow$ $\mathbb{R}$ of the problem

$$
\left\{\begin{array}{l}
\left(-D_{11}\left(\partial_{\theta}\right)^{2}-D_{22}\left(\partial_{\xi}\right)^{2}-D_{33}\left(\partial_{\eta}\right)^{2}+\alpha\right) R_{\alpha, D}^{\infty}=\alpha \delta_{e}, \\
R_{\alpha, D}^{\infty}(\cdot, \cdot, \theta) \rightarrow 0 \text { uniformly on compacta as }|\theta| \rightarrow \infty, \\
R_{\alpha, D}^{\infty} \in \mathbb{L}_{1}\left(\mathbb{R}^{3}\right)
\end{array}\right.
$$

is given by $R_{\alpha, D}^{\infty}(x, y, \theta)=\mathcal{F}^{-1}\left[\left(\omega_{x}, \omega_{y}\right) \mapsto \hat{R}_{\alpha, D}^{\infty}\left(\omega_{x}, \omega_{y}, \theta\right)\right](x, y)$, where

$$
\begin{aligned}
& \hat{R}_{\alpha, D}^{\infty}\left(\omega_{x}, \omega_{y}, \theta\right)=\frac{-\alpha}{4 \pi D_{11} W_{a, q}} \\
& \times\left[\mathrm{me}_{\nu}\left(\varphi, \frac{\left(D_{22}-D_{33}\right) \rho^{2}}{4 D_{11}}\right) \mathrm{me}_{-\nu}\left(\varphi-\theta, \frac{\left(D_{22}-D_{33}\right) \rho^{2}}{4 D_{11}}\right) \mathrm{u}(\theta)\right. \\
& \left.\quad+\mathrm{me}_{-\nu}\left(\varphi, \frac{\left(D_{22}-D_{33}\right) \rho^{2}}{4 D_{11}}\right) \mathrm{me}_{\nu}\left(\varphi-\theta, \frac{\left(D_{22}-D_{33}\right) \rho^{2}}{4 D_{11}}\right) \mathrm{u}(-\theta)\right]
\end{aligned}
$$

with $\boldsymbol{\omega}=(\rho \cos \phi, \rho \sin \phi)$, where $\theta \mapsto \mathrm{u}(\theta)$ denotes the unit step function, which is given by $\mathrm{u}(\theta)=1$ if $\theta>0, \mathrm{u}(\theta)=0$ if $\theta<0$. The Floquet exponent equals $\nu\left(\frac{-\left(\alpha+(1 / 2)\left(D_{22}+D_{33}\right) \rho^{2}\right)}{D_{11}}, \frac{\left(D_{22}-D_{33}\right) \rho^{2}}{4 D_{11}}\right)$ and $W_{a, q}=\mathrm{ce}_{\nu}(0, q) \mathrm{se}_{\nu}^{\prime}(0, q)$ equals the Wronskian of $\operatorname{ce}(\cdot, q)$ and $\operatorname{se}(\cdot, q)$ with $a=\frac{-\left(\alpha+(1 / 2)\left(D_{22}+D_{33}\right) \rho^{2}\right)}{D_{11}}$ and $q=\frac{\left(D_{22}-D_{33}\right) \rho^{2}}{4 D_{11}}$.

In case $D_{22}=D_{33}$ (which follows by taking the limit $D_{22} \rightarrow D_{33}$ in (5.7)) we have

$$
\hat{R}_{\alpha, D}^{\infty}(\boldsymbol{\omega}, \theta)=\frac{\alpha e^{-\sqrt{\frac{\alpha+D_{22} \rho^{2}}{D_{11}}}|\theta|}}{4 \pi \sqrt{D_{11}} \sqrt{D_{22} \rho^{2}+\alpha}}, \quad \rho=\|\boldsymbol{\omega}\|, D_{22}=D_{33},
$$

which yields (for $D_{22}=D_{33}$ ):

$$
\begin{aligned}
& K_{s}^{D ; \infty}(\mathbf{x}, \theta)=\frac{1}{\sqrt{D_{11}} D_{22}} \frac{1}{(4 \pi s)^{\frac{3}{2}}} e^{-\frac{\theta^{2}}{D_{11}}+\frac{r^{2}}{D_{22}}}, \quad r=\|\mathbf{x}\|, D_{22}=D_{33}, \\
& R_{\alpha, D}^{\infty}(\mathbf{x}, \theta)=\frac{\alpha}{4 \pi} \frac{1}{\sqrt{D_{11}} D_{22}} \frac{e^{-\sqrt{\alpha} \frac{\theta^{2}}{D_{11}}+\frac{r^{2}}{D_{22}}}}{\sqrt{\frac{\theta^{2}}{D_{11}}+\frac{r^{2}}{D_{22}}}} .
\end{aligned}
$$

Proof. We apply a Fourier transform with respect to $\mathbb{R}^{2}$ only, which yields

$$
\begin{array}{ll}
\left(D_{22}\left(\partial_{\xi}\right)^{2}+D_{33}\left(\partial_{\eta}\right)^{2}+D_{11}\left(\partial_{\theta}\right)^{2}-\alpha I\right) R_{\alpha, D}^{\infty}=-\alpha \delta_{e} & \Leftrightarrow \\
\left(-D_{22} \rho^{2} \cos ^{2}(\varphi-\theta)-D_{33} \rho^{2} \sin ^{2}(\varphi-\theta)+D_{11}\left(\partial_{\theta}\right)^{2}-\alpha I\right) \hat{R}_{\alpha, D}^{\infty}=-\frac{\alpha}{2 \pi} \delta_{0}^{\theta} & \Leftrightarrow \\
\left(-D_{33} \rho^{2}+\left(D_{33}-D_{22}\right) \rho^{2} \cos ^{2}(\varphi-\theta)+D_{11}\left(\partial_{\theta}\right)^{2}-\alpha I\right) \hat{R}_{\alpha, D}^{\infty}=-\frac{\alpha}{2 \pi} \delta_{0}^{\theta} & \Leftrightarrow \\
\left(\left(\partial_{\theta}\right)^{2}+a I-2 q \cos (2(\phi-\theta))\right) \hat{R}_{\alpha, D}^{\infty}=-\frac{\alpha}{2 \pi D_{11}} \delta_{0}^{\theta}, &
\end{array}
$$

\footnotetext{
${ }^{7}$ There exist several definitions of Mathieu solutions (for an overview see [1, p.744, Table 20.10]), each with different normalizations. In this article we always follow the consistent conventions by Meixner and Schaefke [4]. However, for example, Mathematica 5.2 chooses an unspecified convention. This requires a slight modification of (5.5); see 2].
} 
where $a=-\left(\frac{\alpha+\left(\rho^{2} / 2\right)\left(D_{22}+D_{33}\right)}{D_{11}}\right)$ and $q=\rho^{2}\left(\frac{D_{22}-D_{33}}{4 D_{11}}\right)$. Now the remainder of the proof is tangential/analogous to our proof of a similar theorem for the contour-completion process [20, Thm. 4.10], so we omit it here. For details see [21, p.16].

Note that if $D_{22}=D_{33}$, the diffusion in the spatial part is isotropic and $\Delta=\partial_{\xi}^{2}+\partial_{\eta}^{2}=$ $\partial_{x}^{2}+\partial_{y}^{2}$ commutes with $\partial_{\theta}^{2}$. Therefore if $D_{22}=D_{33}$, then left-invariant diffusion on $\mathbb{R}^{2} \times \mathbb{T}$ (with direct product), left-invariant diffusion on $\mathbb{R}^{2} \rtimes \mathbb{T}$ (with semi-direct product), and thereby the kernels (5.8) coincide with the Green's functions for anisotropic diffusion on $\mathbb{R}^{3}$. We have employed this fact in [29] in order to generalize fast Gaussian derivatives on images with separable Gaussian kernels to fast Gaussian derivatives on orientation scores. For details and implementation, see [30, ch:5.2].

Finally, analogously to the contour-completion case [20, ch:4.2.1], we stress that we can expand the exact Green's function $R_{\alpha, D_{11}}$ as an infinite sum over $2 \pi$-shifts of the solution $R_{\alpha, D_{11}}^{\infty}$ for the unbounded case, i.e.

$$
R_{\alpha, D}\left(x, y, e^{i \theta}\right)=\lim _{N \rightarrow \infty} \sum_{k=-N}^{N} R_{\alpha, D}^{\infty}(x, y, \theta-2 k \pi) .
$$

We stress that the rapidly decaying sum in (5.10) can be computed explicitly by means of the Floquet theorem, i.e. (5.6), and the geometrical series $\sum_{k=0}^{\infty} r^{k}=\frac{1}{1-r}$ for $r=e^{i \nu}$ with $r=\left|e^{i \nu}\right|<1$ since the imaginary part of $\nu=\nu(a, q)$ is positive. By straightforward computations this yields the following result.

Theorem 5.3. Let $\alpha, D_{11}, D_{22}>0$ and $D_{33} \geq 0$. Then the solution $R_{\alpha, D}: S E(2) \rightarrow \mathbb{R}$ of the problem

$$
\left\{\begin{array}{l}
\left(-D_{11}\left(\partial_{\theta}\right)^{2}-D_{22}\left(\partial_{\xi}\right)^{2}-D_{33}\left(\partial_{\eta}\right)^{2}+\alpha\right) R_{\alpha, D}=\alpha \delta_{e} \\
R_{\alpha, D}(\cdot, \cdot, \theta+2 k \pi)=R_{\alpha, D}(\cdot, \cdot, \theta) \text { for all } k \in \mathbb{Z}, \\
R_{\alpha, D} \in \mathbb{L}_{1}(S E(2))
\end{array}\right.
$$

is given by $R_{\alpha, D}(\mathbf{x}, \theta)=\sum_{k \in \mathbb{Z}} R_{\alpha, D}^{\infty}(\mathbf{x}, \theta+2 k \pi)$, the right-hand side of which can be calculated using Floquet's theorem and (5.7) yielding for $D_{33}<D_{22}$ :

$$
\begin{aligned}
& {\left[\mathcal{F} R_{\alpha, D}(\cdot, \theta)\right](\boldsymbol{\omega})=\frac{\alpha}{4 \pi D_{11} \mathrm{ce}_{\nu}(0, q) \mathrm{se}_{\nu}^{\prime}(0, q)}} \\
& \times\left\{\left(-\cot (\nu \pi)\left(\operatorname{ce}_{\nu}(\varphi, q) \operatorname{se}_{\nu}(\varphi-\theta, q)+\operatorname{se}_{\nu}(\varphi, q) \operatorname{se}_{\nu}(\varphi-\theta, q)\right)\right.\right. \\
& \left.\quad+\operatorname{ce}_{\nu}(\varphi, q) \operatorname{se}_{\nu}(\varphi-\theta, q)-\operatorname{se}_{\nu}(\varphi, q) \operatorname{ce}_{\nu}(\varphi-\theta, q)\right) \mathrm{u}(\theta) \\
& +\left(-\cot (\nu \pi)\left(\operatorname{ce}_{\nu}(\varphi, q) \operatorname{ce}_{\nu}(\varphi-\theta, q)-\operatorname{se}_{\nu}(\varphi, q) \operatorname{se}_{\nu}(\varphi-\theta, q)\right)\right. \\
& \left.\left.\quad+\operatorname{ce}_{\nu}(\varphi, q) \operatorname{se}_{\nu}(\varphi-\theta, q)+\operatorname{se}_{\nu}(\varphi, q) \operatorname{ce}_{\nu}(\varphi-\theta, q)\right) \mathrm{u}(-\theta)\right\}
\end{aligned}
$$

with $q=\frac{\left(D_{22}-D_{33}\right) \rho^{2}}{4 D_{11}}, \boldsymbol{\omega}=(\rho \cos \varphi, \rho \sin \varphi)$ and the Floquet exponent $\nu=\nu(a, q)$, $a=-\frac{\alpha+(1 / 2)\left(D_{22}-D_{33}\right) \rho^{2}}{D_{11}}$ and where $\theta \mapsto \mathrm{u}(\theta)$ denotes the unit step function, which is given by $\mathrm{u}(\theta)=1$ if $\theta>0, \mathrm{u}(\theta)=0$ if $\theta<0$.

The results in the preceding theory on the resolvent Green's function of the contourenhancement process can be set in a variational formulation, similar to the variational formulation in [10] (where $D_{33}=0$ ). 
Corollary 5.4. Let $U \in \mathbb{L}_{2}(S E(2))$ and $\alpha, D_{11}, D_{22}>0, D_{33} \geq 0$. Then the unique solution of the variational problem

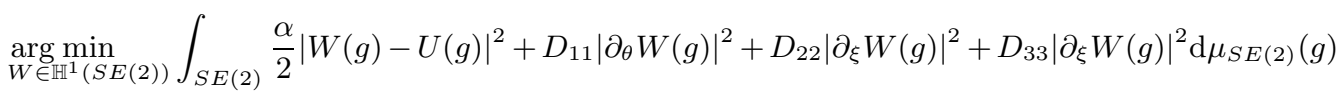

is given by $W(g)=\left(R_{\alpha, D} *_{S E(2)} U\right)(g)=\int_{S E(2)} R_{\alpha, D}\left(h^{-1} g\right) U(h) \mathrm{d} \mu_{S E(2)}(h)$, where the Green's function $R_{\alpha, D}: S E(2) \rightarrow \mathbb{R}^{+}$is explicitly given in Theorem 5.3 .

For a proof see [21, p.18].

5.2. The Heisenberg approximations of the heat kernels on $S E(2)$. If we apply a firstorder approximation $\cos \theta \approx 1$ and $\sin \theta \approx \theta$ the left-invariant vector fields $\left\{\mathcal{A}_{1}, \mathcal{A}_{2}, \mathcal{A}_{3}\right\}$ are approximated by

$$
\hat{A}_{1}=\partial_{\theta}, \hat{A}_{2}=\partial_{x}+\theta \partial_{y}, \hat{A}_{5}=-\theta \partial_{x}+\partial_{y}
$$

which are left-invariant vector fields in a 5-dimensional nilpotent Lie algebra (adding the directions $\hat{A}_{3}=\partial_{y}, \hat{A}_{4}=\partial_{x}$ ) of Heisenberg type. In our previous related work 20] we used this replacement to explicitly derive more tangible Green's functions which are surprisingly good approximations of the exact Green's functions of the direction process. In fact the replacements $\mathcal{A}_{1} \rightarrow \hat{A}_{1}$ and $\mathcal{A}_{2} \rightarrow \hat{A}_{2}$ (while considering the case $D_{33}=0$ ) will provide Green's functions on the group of positions and velocities rather than Green's functions on the group of positions and orientations; see [53, App. C]. The Lie algebra of this 3-dimensional subgroup $H_{3}$ is spanned by

$$
\left\{\hat{A}_{1}=\partial_{\theta}, \hat{A}_{2}=\partial_{x}+\theta \partial_{y}, \hat{A}_{3}=\left[\hat{A}_{1}, \hat{A}_{2}\right]=\partial_{y}\right\} .
$$

Later in Section 5.4 we will underpin the intuitive replacements $\mathcal{A}_{1} \rightarrow \hat{A}_{1}$ and $\mathcal{A}_{2} \rightarrow \hat{A}_{2}$ by decent mathematics, following the method of contraction as described in [52].

Here we will derive the approximative Green's functions for contour enhancement, which coincide, up to coordinate transformation, with the heat kernels on $H_{3}$. In the case of contour completion we had the interesting situation that the approximative leftinvariant vector field $\hat{A}_{2}=\partial_{x}+\theta \partial_{y}$ together with the diffusion generator $\left(\partial_{\theta}\right)^{2}$ and the identity operator $I$ and all commutators form an 8-dimensional nilpotent Lie algebra spanned by $\left\{I, \partial_{x}, \partial_{\theta}, \partial_{y}, \theta \partial_{y}, \partial_{\theta}^{2}, \partial_{\theta} \partial_{y}, \partial_{y}^{2}\right\}$. From this observation and [55, Theorem 3.18 .11 p.243] it follows that the approximative Green's functions are given by

$$
\begin{aligned}
\bar{K}_{s}^{D_{11}, a_{2}=1}(x, y, \theta) & =\delta(x-s) \frac{\sqrt{3}}{2 D_{11} \pi x^{2}} e^{-\frac{3(x \theta-2 y)^{2}+x^{2}\left(\theta-\kappa_{0} x\right)^{2}}{4 x^{3} D_{11}}}, \\
\bar{R}_{\alpha, D_{11}, a_{2}=1}(x, y, \theta) & =\alpha \frac{\sqrt{3}}{2 D_{11} \pi x^{2}} e^{-\alpha x} e^{-\frac{3(x \theta-2 y)^{2}+x^{2}\left(\theta-\kappa_{0} x\right)^{2}}{4 x^{3} D_{11}}} \mathrm{u}(\mathrm{x}),
\end{aligned}
$$

where $\mathrm{u}$ denotes the 1D Heaviside/unit step function. However, this efficient technique cannot be applied to the diffusion case (contour enhancement), since the commutators of the separate diffusion generators provide infinitely many directions. Therefore we follow [10] and apply a coordinate transformation

$$
\bar{K}_{s}^{D_{11}, D_{22}}(x, y, \theta)=\tilde{K}_{s}\left(x^{\prime}, \omega^{\prime}, t^{\prime}\right)=\tilde{K}_{s}\left(\frac{x}{\sqrt{2 D_{22}}}, \frac{\theta}{\sqrt{2 D_{11}}}, \frac{2\left(y-\frac{x \theta}{2}\right)}{\sqrt{D_{11} D_{22}}}\right),
$$


where we note that $\partial_{s} \bar{K}_{s}^{D_{11}, D_{22}}=\left(D_{11} \partial_{\theta}^{2}+D_{22}\left(\partial_{x}+\theta \partial_{y}\right)^{2}\right) \bar{K}_{s}^{D_{11}, D_{22}}$ if and only if $\partial_{s} \tilde{K}_{s}=\frac{1}{2}\left(\left(\partial_{\omega^{\prime}}-2 x^{\prime} \partial_{t^{\prime}}\right)^{2}+\left(\partial_{x^{\prime}}+2 \omega^{\prime} \partial_{t^{\prime}}\right)^{2}\right) \tilde{K}_{s}=\frac{1}{2} \Delta_{K} \tilde{K}_{s}$, which provides us the leftinvariant evolution equation on the usual Heisenberg group $H_{3}$ generated by Kohn's Laplacian, 32. So now we can translate well-known results on harmonic analysis on $\mathrm{H}_{3}$ to the diffusion equation of the contour-enhancement process. For example the heat kernel and fundamental solution on $H_{3}$ are well known [32. The heat kernel equals

$$
\tilde{K}_{s}\left(x^{\prime}, \omega^{\prime}, t^{\prime}\right)=\frac{1}{(2 \pi s)^{2}} \int_{\mathbb{R}} \frac{2 \tau}{\sinh (2 \tau)} \cos \left(\frac{\tau t^{\prime}}{s}\right) e^{-\frac{\left(\frac{\left(x^{\prime}\right)^{2}}{s}+\frac{\left(\omega^{\prime}\right)^{2}}{s}\right) \tau}{\tanh (2 \tau)}} \mathrm{d} \tau
$$

and as a result by (5.14) we obtain 8 the following Heisenberg-type approximation of the Green's function and corresponding resolvent (for infinite expected lifetime, (3.15)):

$$
\begin{aligned}
& \bar{K}_{s}^{D_{11}, D_{22}}(x, y, \theta)=\frac{1}{2 D_{11} D_{22}} \tilde{K}_{s}\left(\frac{x}{\sqrt{2 D_{22}}}, \frac{\theta}{\sqrt{2 D_{11}}}, \frac{2\left(y-\frac{x \theta}{2}\right)}{\sqrt{D_{11} D_{22}}}\right) \\
& \quad=\frac{1}{8 D_{11} D_{22} \pi^{2} s^{2}} \int_{\mathbb{R}} \frac{2 \tau}{\sinh (2 \tau)} \cos \left(\frac{2 \tau\left(y-\frac{x \theta}{2}\right)}{s \sqrt{D_{11} D_{22}}}\right) e^{-\frac{\left(\frac{x^{2}}{s D_{22}}+\frac{\theta^{2}}{s D_{11}}\right) \tau}{2 \tanh (2 \tau)}} \mathrm{d} \tau \\
& \text { and } \lim _{\alpha \rightarrow 0} \alpha^{-1} \bar{R}_{\alpha, D_{11}, D_{22}}(x, y, \theta)=\frac{1}{4 \pi D_{11} D_{22}} \frac{1}{\sqrt{\frac{1}{16}\left(\frac{x^{2}}{D_{22}}+\frac{\theta^{2}}{D_{11}}\right)^{2}+\frac{\left(y-\frac{1}{2} x \theta\right)^{2}}{D_{11} D_{22}}}} .
\end{aligned}
$$

The resolvent Green's function $\lim _{\alpha \rightarrow 0} \alpha^{-1} \bar{R}_{\alpha, D_{11}, D_{22}}$ follows by the fundamental solution on $H_{3}, 32$ and the coordinate transform (5.14).

For a detailed derivation of (5.15), (5.16), we refer to our earlier work [19, pp. 6-8], which mainly follows derivations by [32] on $\mathrm{H}_{3}$, where we systematically applied (5.14) to relate the coordinates of the first kind to coordinates of the second kind on $\mathrm{H}_{3}$. Here we also provide the corresponding resolvent kernel with finite expected lifetime $\alpha^{-1}$ : $\tilde{R}_{\alpha}\left(x^{\prime}, \omega^{\prime}, t^{\prime}\right)=\alpha \int_{\mathbb{R}^{+}} \tilde{K}_{s}^{\mathbf{D}}\left(x^{\prime}, \omega^{\prime}, t^{\prime}\right) e^{-\alpha s} \mathrm{~d} s$, which is given by

$$
\begin{aligned}
\bar{R}_{\alpha}(x, y, \theta) & =\tilde{R}_{\alpha}\left(x^{\prime}, \omega^{\prime}, t^{\prime}\right) \text { with }\left(x^{\prime}, \omega^{\prime}, t^{\prime}\right)=\left(\frac{x}{\sqrt{2 D_{22} 2}}, \frac{\theta}{\sqrt{2 D_{11}}}, \frac{2\left(y-\frac{x \theta}{2}\right)}{\sqrt{D_{11} D_{22}}}\right) \\
& =\frac{2 \alpha \sqrt{\alpha}}{\pi^{2}} \int_{0}^{\infty} \frac{\tau}{\sinh 2 \tau} \operatorname{Re}\left(\frac{k_{1}\left(2 \sqrt{\alpha} \sqrt{\frac{2 \tau}{\tanh 2 \tau}\left(\frac{\left(x^{\prime}\right)^{2}}{D_{11}}+\frac{\left(\omega^{\prime}\right) 2^{2}}{D_{22}}-\frac{2 i \tau t^{\prime}}{\sqrt{D_{11} D_{22}}}\right)}\right.}{\sqrt{\frac{2 \tau}{\tanh 2 \tau}\left(\frac{\left(x^{\prime}\right)^{2}}{D_{11}}+\frac{\omega^{2}}{D_{22}}\right)-\frac{2 i \tau t^{\prime}}{\sqrt{D_{11} D_{22}}}}}\right) \mathrm{d} \tau,
\end{aligned}
$$

where $k_{1}$ is the first-order Bessel K-function. Formulae (5.17) and (5.16) are somewhat cumbersome if it comes to fast computations in practice. Later, in Section 5.4 we shall derive asymptotic formulas for these kernels that are more tangible from a practical point of view. However, the resolvent kernel with infinite lifetime (5.16) is much simpler and follows by taking the limit $\alpha \rightarrow 0$ in (5.17) and substitution $v=\cosh (2 \tau)$.

Figure 7 shows illustrations of both the exact resolvent Green's function $R_{\alpha, D_{11}, D_{22}}$ and its approximation $\bar{R}_{\alpha, D_{11}, D_{22}}$, which coincides with the resolvent kernel on $H_{3}$ :

$$
\bar{R}_{\alpha, D_{11}, D_{22}}=\left(D_{11} \hat{A}_{1}^{2}+D_{22} \hat{A}_{2}^{2}-\alpha I\right)^{-1} \delta_{e} .
$$

8 Note that our approximation of the Green's function on the Euclidean motion group does not coincide with the formula by Citti in 10 . 

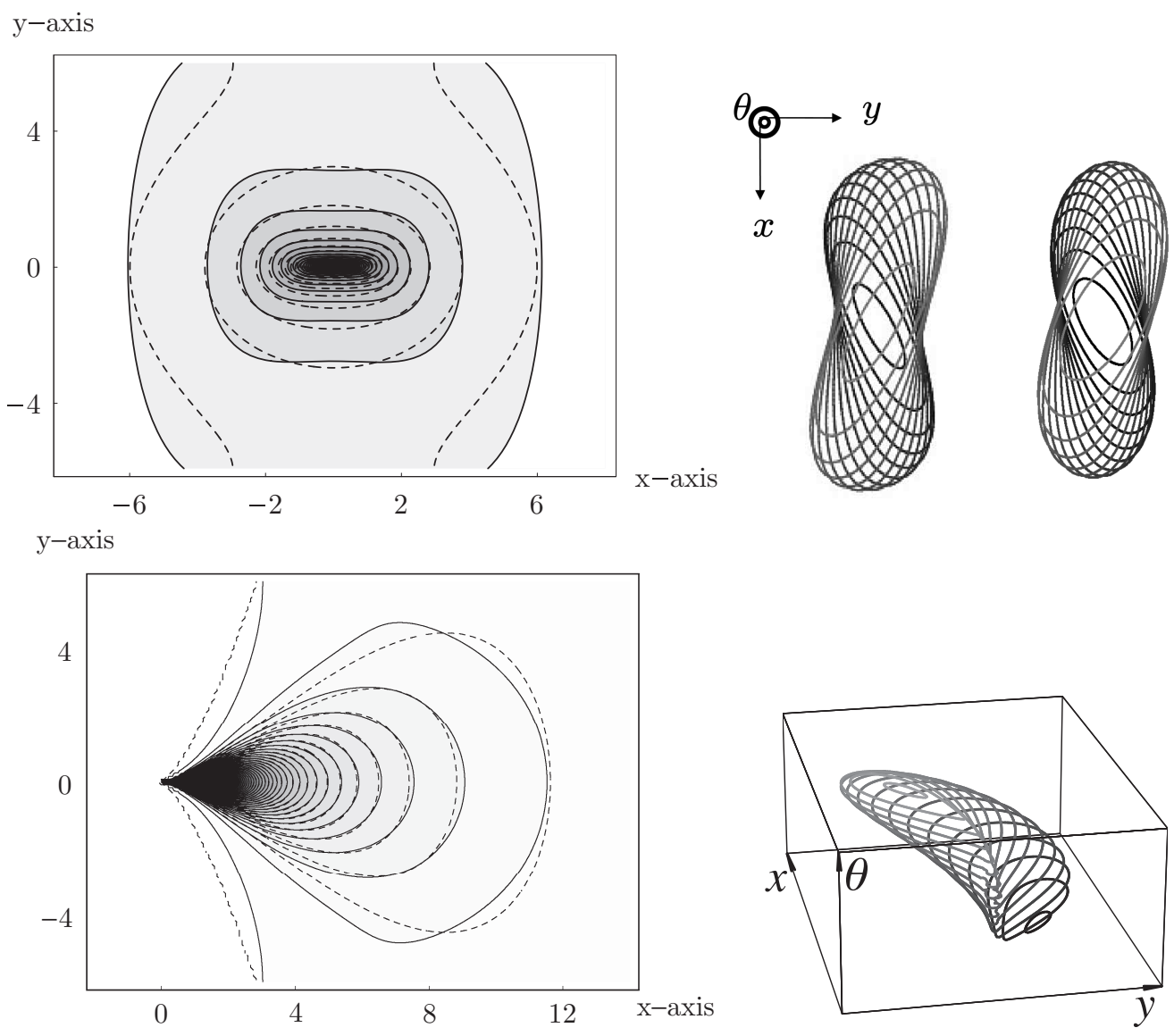

FIG. 7. Top row, left: A comparison between the exact Green's function $R_{\alpha, D_{11}, D_{22}}$ of the resolvent diffusion process $\alpha=\frac{1}{30}, D_{11}=0.1$, $D_{22}=0.5$ on $S E(2)$ in Theorem 5.3 and the approximate Green's function $\bar{R}_{\alpha, D_{11}, D_{22}}$ (5.18) (iso-contours in dashed lines) of the corresponding resolvent process with infinite lifetime $(\alpha \rightarrow 0)$ on $S E(2)$ given by (5.16). Here both distributions are integrated over the torus, yielding the $x y$-marginals. Top row right: $3 \mathrm{D}$-view on a stack of isocontours (intersections by fixed $\theta$-planes) in $S E(2)$ (left: approximation $\bar{R}_{\alpha, D_{11}, D_{22}}=C$, right: exact $R_{\alpha, D_{11}, D_{22}}(\cdot, \cdot, \theta)=C$ ) viewed along the $\theta$-direction. Bottom row: a comparison of the level curves of the marginals of $\bar{R}_{\alpha, D_{11}}$ (given by (5.13) ) and the exact Green's function of the direction process $R_{\alpha, D_{11}, a_{2}=1}$ given in [20]. Right: 3 D-view on a stack of iso-contours $R_{\alpha, D_{11}, a_{2}=1}(\cdot, \cdot, \theta)=C$ in $S E(2)$. Left: comparison of the $x y$-marginals, where again dashed lines denote the level sets of the approximation $\bar{R}_{\alpha, D_{11}, a_{2}=1}$. The small difference is best seen in the iso-contours close to zero. In both cases the typical difference between the dashed (approximation) and nondashed iso-contours (exact) contours is due to the fact that random walkers of the approximation processes (such as (5.21) ) on $H_{3}$ must move forward in the initial $x$-direction and thereby, in contrast to the random walkers of the exact processes on $S E(2)$ (such as (3.12)), do not turn in the negative $x$-direction. 
5.3. The Hörmander condition and the underlying stochastics of the Heisenberg approximation of the diffusion process on $S E(2)$. In this subsection we will address two issues. First we derive necessary and sufficient conditions on the convection and diffusion parameters, respectively $\mathbf{a}=\left(a_{1}, a_{2}, a_{3}\right)$ and $D=\left[D_{i j}\right]$, in order to get smooth Green's functions of the left-invariant convection-diffusions (3.6) with generator (3.5). It turns out that $D$ need not be strictly positive, as for example in the case of the contourenhancement process we have $\mathbf{a}=(0,0,0)$ and $D=\operatorname{diag}\left\{D_{11}, D_{22}, 0\right\}$ and in the case of the direction process we even have $\mathbf{a}=(0,1,0)$ and $D=\operatorname{diag}\left\{D_{11}, 0,0\right\}$. By the Hörmander theorem [36] the noncommutative nature of $S E(2)$, in certain cases, takes care of missing directions in the diffusion matrix (i.e., directions in the null-space of $D)$. Secondly, we would like to get a stochastic grip on the induced smoothing in these missing directions.

Consider the contour-enhancement kernel

$$
K_{s}^{D_{11}=1, D_{22}=1,0}(x, y, \theta)=\left(e_{S E(2)}^{t\left(\partial_{\xi}^{2}+\partial_{\theta}^{2}\right)} \delta_{e}\right)(x, y, \theta)
$$

on the group $S E(2)$ and its approximation

$$
\tilde{K}_{s}^{1,1,0}(x, y, \theta)=\left(e_{H_{3}}^{t\left(\left(\partial_{x}+\theta \partial_{y}\right)^{2}+\partial_{\theta}^{2}\right)} \delta_{e}\right)(x, y, \theta)
$$

on the group $H_{3}$. Both kernels are nonsingular and smooth in all directions, whereas the corresponding kernel on the commutative group $\left(\mathbb{R}^{3},+\right)$, given by $\left(e_{\mathbb{R}^{2}}^{t\left(\partial_{x}^{2}+\partial_{\theta}^{2}\right)} \delta_{0}^{x} \otimes \delta_{0}^{y} \otimes\right.$ $\left.\delta_{0}^{\theta}\right)(x, y, \theta)=G_{t}^{d=1}(x) G_{t}^{d=1}(\theta) \delta_{0}^{y}$, is the singular Green's function of Brownian motion in the $(x, \theta)$-plane in $\mathbb{R}^{3}$. Throughout this subsection we shall restrict ourselves to the Heisenberg approximation of contour enhancement to illustrate our issues. Regarding our second issue we show how the indirect smoothing in the $\partial_{y}$-direction relates to a random variable that depends on random variables related to the direct smoothing in the $\partial_{x}$ and $\partial_{\theta}$ directions. First we will formulate the Hörmander theorem.

A differential operator $L$ defined on a manifold $M$ of dimension $n \in \mathbb{N}, n<\infty$ is called hypo-elliptic if for all distributions $f$ defined on an open subset of $M$ such that $L f$ is $C^{\infty}$ (smooth), $f$ must also be $C^{\infty}$. In his paper [36, Hörmander presented a sufficient and essentially necessary condition for an operator of the type $L=c+X_{0}+\sum_{i=1}^{r}\left(X_{i}\right)^{2}$, $r \leq n$, where $\left\{X_{i}\right\}$ are vector fields on $M$, to be hypo-elliptic. This condition, which we shall refer to as the Hörmander condition, is that among the set

$$
\left\{X_{j_{1}},\left[X_{j_{1}}, X_{j_{2}}\right],\left[X_{j_{1}},\left[X_{j_{2}}, X_{j_{3}}\right]\right], \ldots,\left[X_{j_{1}},\left[X_{j_{2}},\left[X_{j_{3}}, \ldots, X_{j_{k}}\right]\right]\right] \ldots \mid j_{i} \in\{0,1, \ldots, r\}\right\}
$$

there exist $n$ elements which are linearly independent at any given point in $M$. Now if $M$ is a Lie group and we restrict ourselves to left-invariant vector fields, then it is sufficient to check whether the vector fields span the tangent space at the unity element. So necessary and sufficient conditions for smooth (resolvent) Green's functions on $S E(2) \backslash\{e\}$ on the diffusion and convection parameters $(D, \mathbf{a})$ in the generator (3.5) of (3.6) for diagonal $D$ are

$$
\{1,3\} \subset\left\{i \mid a_{i} \neq 0 \vee D_{i i} \neq 0\right\} \quad \vee \quad\{1,2\} \subset\left\{i \mid a_{i} \neq 0 \vee D_{i i} \neq 0\right\} .
$$

If we apply this theorem to the forward Kolmogorov equation of the direction process, then we see that the Hörmander condition is satisfied since we have $M=S E(2) \times \mathbb{R}^{+}$, 
$X_{0}=-\partial_{s}-\partial_{\xi}, X_{1}=\partial_{\theta}$ and we have

$\operatorname{dim} \operatorname{span}\left\{-\partial_{s}-\partial_{\xi}, \partial_{\theta},\left[\partial_{\theta},-\partial_{s}-\partial_{\xi}\right],\left[\partial_{\theta},\left[\partial_{\theta},-\partial_{s}-\partial_{\xi}\right]\right]\right\}=\operatorname{dim} \operatorname{span}\left\{\partial_{s}, \partial_{\theta}, \partial_{\xi}, \partial_{\eta}\right\}=4$ and indeed the Green's function of Mumford's direction process is infinitely differentiable on $S E(2)$; see [20]. Similarly the Green's function of the resolvent direction process determined by $L R=\delta_{e}$, with $L=-\partial_{\xi}+D_{11}\left(\partial_{\theta}\right)^{2}-\gamma I$, is infinitely differentiable on $S E(2) \backslash\{e\}$; for explicit formulas, see [20]. To this end set $M=S E(2)$ and note that

$$
\operatorname{span}\left\{\partial_{\theta},\left[\partial_{\theta}, \partial_{\xi}\right],\left[\partial_{\theta},\left[\partial_{\theta}, \partial_{\xi}\right]\right]\right\}=\operatorname{span}\left\{\partial_{\theta}, \partial_{\xi}, \partial_{\eta}\right\}=\mathcal{L}(S E(2)) .
$$

However, in the case of the direction process, the Heisenberg approximation of the timedependent Green's function (5.13) is singular and indeed

$$
\operatorname{dim} \operatorname{span}\left\{-\partial_{s}-\partial_{x}-\theta \partial_{y}, \partial_{\theta}, \partial_{y}\right\}=3<4 .
$$

Fortunately, this discrepancy between the Heisenberg approximation and the exact case does not take place in the contour-enhancement processes, where we have both

$$
\operatorname{dim} \operatorname{span}\left\{-\partial_{s}, \partial_{x}+\theta \partial_{y}, \partial_{\theta}, \partial_{y}\right\}=4 \text { and } \operatorname{dim} \operatorname{span}\left\{\partial_{s}, \partial_{\theta}, \partial_{\xi}, \partial_{\eta}\right\}=4
$$

Now we consider our second issue:

"Can we get stochastic insight in the induced smoothing in the remaining directions in the diffusion processes (3.6) on $S E(2)$ generated by hypo-elliptic operators, of the type (3.5), that are not elliptic?"

Consider to this end the heat kernel on the 3D Heisenberg group $H_{3}$, recall (5.15), which is smooth in all directions, despite the fact that diffusion is only done in the $\partial_{x}+2 \omega \partial_{t}$ and $\partial_{\omega}-2 x \partial_{t}$ directions. Here, the induced smoothness in the $t$-direction has an elegant stochastic interpretation. As shown in [32, the underlying stochastic process (with the diffusion equation on $\mathrm{H}_{3}$ as the forward Kolmogorov equation) is given by

$$
\left\{\begin{array}{l}
Z(s)=X(s)+i W(s)=Z_{0}+\varepsilon \sqrt{s}, \varepsilon \sim \mathcal{N}(0,1), \\
T(s)=2 \int_{0}^{s} W \mathrm{~d} X-X \mathrm{~d} W, s>0
\end{array}\right.
$$

so the random variable $Z=X+i W$ is a Brownian motion in the complex plane and the random variable $T(s)$ measures the deviation from a sample path with respect to a straight path $Z(s)=Z_{0}+s\left(Z(s)-Z_{0}\right)$ by means of the stochastic integral $T(s)=$ $2 \int_{0}^{s} W \mathrm{~d} X-X \mathrm{~d} W$. To this end we note that for $9 \mapsto(x(s), \omega(s)) \in C^{\infty}\left(\mathbb{R}^{+}, \mathbb{R}^{2}\right)$ such that the straight line from $X_{0}$ to $X(s)$ followed by the inverse path encloses an oriented surface $\Omega \in \mathbb{R}^{2}$, we have by Stokes' theorem that

$$
2 \mu(\Omega)=-\int_{0}^{s}\left(-X^{\prime}(t) W(t)+X(t) W^{\prime}(t)\right) \mathrm{d} t+0=\int_{0}^{s} W \mathrm{~d} X-X \mathrm{~d} W .
$$

Now by the coordinate transformation (5.14) we deduce that the underlying stochastic process of the Heisenberg approximation of the diffusion process on $S E(2)$ is given by

$$
\left\{\begin{array}{l}
X(s)+i \Theta(s)=X(0)+i \Theta(0)+\sqrt{s}\left(\epsilon_{x}+i \epsilon_{\theta}\right), \\
Y(s)=Y(0)-\frac{X(0) \Theta(0)}{2}+\frac{X(s) \Theta(s)}{2}+\frac{1}{2} \int_{0}^{s} \Theta \mathrm{d} X-X \mathrm{~d} \Theta,
\end{array}\right.
$$

${ }^{9} \mathrm{~A}$ Brownian motion is a.e. not differentiable in the classical sense, nor does the integral in (5.20) make sense in classical integration theory. 

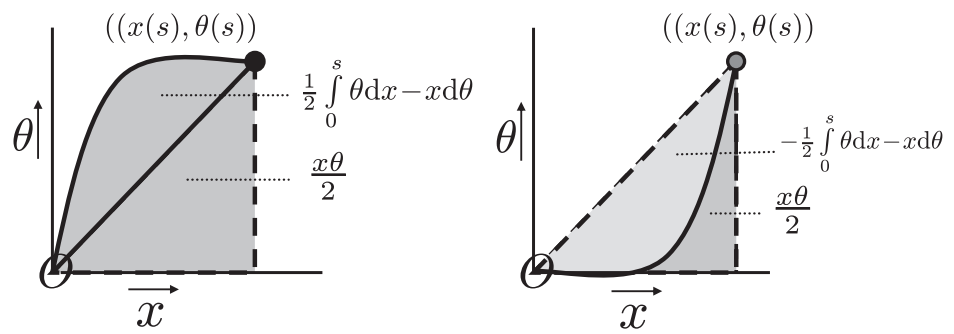

FIG. 8. Stochastic interpretation of the "indirect smoothing" (by means of the commutators) within the Hörmander condition of the diffusion generator of the Heisenberg approximation. See (5.21). The left-invariant diffusion on $H_{3}$ generated only by $\hat{A}_{1}=\partial_{\theta}$ and $\hat{A}_{2}=$ $\partial_{x}+\theta \partial_{y}$ takes place along the corresponding exponential curves of $H_{3}$. Along these curves one has $\frac{d y}{d x}=\theta$ and thereby $\mathrm{d}\left(y-\frac{x \theta}{2}\right)=$ $\frac{1}{2}(\theta \mathrm{d} x-x \mathrm{~d} \theta)$ and the random variable $Y(s)$ (determined by $X$ and $\Theta)$ can be written as the stochastic integral $Y(s)=Y(0)-\frac{X(0) \Theta(0)}{2}+$ $\frac{X(s) \Theta(s)}{2}+\frac{1}{2} \int_{0}^{s} \Theta \mathrm{d} X-X \mathrm{~d} \Theta$ and measures the surface area between a path and the $x$-axis.

with the random variables $\epsilon_{x} \sim \mathcal{N}\left(0,2 D_{11}\right), \epsilon_{\theta} \sim \mathcal{N}\left(0,2 D_{22}\right)$, which provides a better understanding of the "indirect smoothing" (by means of the commutators) within the Hörmander condition of the Heisenberg approximation of the contour-enhancement process on $S E(2)$ : the indirect smoothing in the $\partial_{y}$-direction is due to the randomness of the variable $Y(s)$ given in (5.21), similar to the fact that the direct smoothing in, respectively, the $\partial_{x}$ and $\partial_{\theta}$ directions is due to the randomness of $X(s)$ and $\Theta(s)$. For the simple geometric meaning of the random variable $Y(s)$, see Figure 8 ,

5.4. Gaussian estimates for a parameterized class of intermediate semigroups. In this section we shall derive, only for the case $D_{33}=0$, a continuum of semigroups between the exact semigroup $U \mapsto K_{s}^{D_{11}, D_{22}} *_{S E(2)} U$ with the kernels $K_{s}^{D_{11}, D_{22}}: S E(2) \rightarrow \mathbb{R}^{+}$

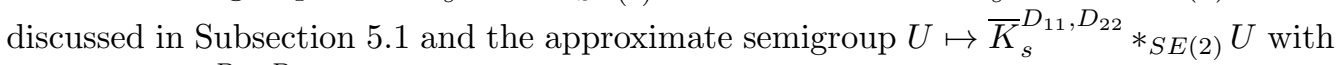
the kernels $\bar{K}_{s}^{D_{11}, D_{22}}: S E(2) \rightarrow \mathbb{R}^{+}$discussed in Subsection 5.2 $s>0$. Furthermore, we shall derive Gaussian estimates for both $K_{s}^{D_{11}, D_{22}}$ and $\bar{K}_{s}^{D_{11}, D_{22}}, s>0$. For the latter case this Gaussian estimate turns out to be rather sharp.

We follow the general work by ter Elst and Robinson [23] on semigroups on Lie groups generated by weighted subcoercive operators. In their general work we consider a particular case by setting the Hilbert space $H=\mathbb{L}_{2}(S E(2))$, the group $G=S E(2)$ and the right-regular representation $\mathcal{U}=\mathcal{R}$. For details, see [21, App.D]. Furthermore we consider the algebraic basis $\left\{\partial_{\theta}=\mathrm{d} \mathcal{R}\left(A_{1}\right), \partial_{\xi}=\mathrm{d} \mathcal{R}\left(A_{2}\right)\right\}$ leading to the following filtration of the Lie algebra:

$$
\mathfrak{g}_{1}=\operatorname{span}\left\{\partial_{\theta}, \partial_{\xi}\right\} \subset \mathfrak{g}_{2}=\operatorname{span}\left\{\partial_{\theta}, \partial_{\xi}, \partial_{\eta}\right\}
$$

so the weights are $w_{1}=1, w_{2}=1$ and $w_{3}=2$. For example $\partial_{\eta}$ has weight 2 since it occurs in $\mathfrak{g}_{2}$ but not in $\mathfrak{g}_{1}$. Now we define the dilations on the Lie algebra and the Lie 
group:

$$
\begin{aligned}
& \gamma_{t}\left(\sum_{i=1}^{3} c^{i} A_{i}\right)=\sum_{i=1}^{3} t^{w_{i}} c^{i} A_{i}, \text { for all } c^{i} \in \mathbb{R} \\
& \tilde{\gamma}_{t}(x, y, \theta)=\left(\frac{x}{t^{w_{2}}}, \frac{y}{t^{w_{3}}}, e^{i \frac{\theta}{t^{w_{1}}}}\right), \quad \text { with } w_{1}=w_{2}=1, w_{3}=2
\end{aligned}
$$

and for $0<t \leq 1$ we define the Lie product $[A, B]_{t}=\gamma_{t}^{-1}\left[\gamma_{t}(A), \gamma_{t}(B)\right]$. Now let $(S E(2))_{t}$ be the simply connected Lie group generated by the Lie algebra $\left(T_{e}(S E(2)),[\cdot, \cdot]_{t}\right)$. The group products on the intermediate groups $(S E(2))_{t \in(0,1]}$ are given by

$$
(x, y, \theta) \cdot{ }_{t}\left(x^{\prime}, y^{\prime}, \theta^{\prime}\right)=\left(x+\cos (\theta t) x^{\prime}-t \sin (\theta t) y^{\prime}, y+\frac{\sin (\theta t)}{t} x^{\prime}+\cos (\theta t) y^{\prime}, \theta+\theta^{\prime}\right) .
$$

The left-invariant vector fields on $(S E(2))_{t}$ are given by $\left.\mathcal{A}_{i}^{t}\right|_{g}=\left(\tilde{\gamma}_{t}^{-1} \circ L_{g} \circ \tilde{\gamma}_{t}\right)_{*} A_{i}$, so

$$
\begin{aligned}
& \left.\mathcal{A}_{1}^{t}\right|_{g}=\frac{1}{t}\left(t \partial_{\theta}\right)=\partial_{\theta} \\
& \left.\mathcal{A}_{2}^{t}\right|_{g}=t\left(\frac{\cos (\theta t)}{t} \partial_{x}+\frac{\sin \theta t}{t^{2}} \partial_{y}\right)=\cos (\theta t) \partial_{x}+\frac{\sin (\theta t)}{t} \partial_{y}, \\
& \left.\mathcal{A}_{3}^{t}\right|_{g}=t^{2}\left(-\frac{\sin (\theta t)}{t} \partial_{x}+\frac{\cos (\theta t)}{t^{2}} \partial_{y}\right)=-t \sin (\theta t) \partial_{x}+\cos (\theta t) \partial_{y} .
\end{aligned}
$$

Now the homogeneous nilpotent contraction Lie algebra equals

$$
(S E(2))_{0}=\lim _{t \downarrow 0}(S E(2))_{t} \equiv H_{3} \quad \text { and }(S E(2))_{t=1} /(\{0\} \times\{0\} \times 2 \pi \mathbb{Z})=S E(2),
$$

with the Lie algebra $\mathcal{L}\left(H_{3}\right)=\mathcal{L}\left((S E(2))_{0}\right)=\operatorname{span}\left\{\partial_{\theta}, \partial_{x}+\theta \partial_{y}, \partial_{y}\right\}=\left\{\hat{A}_{1}, \hat{A}_{2}, \hat{A}_{3}\right\}$, where we recall (5.12). Note that $\mathcal{L}(S E(2))=\mathcal{L}\left((S E(2))_{t=1}\right)=\left\{\partial_{\theta}, \partial_{\xi}, \partial_{\eta}\right\}$.

So we have derived a continuum of holomorphic semigroups between the exact case and its Heisenberg approximation with rapidly decaying kernels $K_{s}^{t} \in \mathbb{L}_{2}\left((S E(2))_{t}\right) \cap$ $\mathbb{L}_{1}\left((S E(2))_{t}\right)$ that satisfy Gaussian estimates $\left|K_{s}^{t}(g)\right| \leq C s^{-2} e^{-b \frac{\left(|g|_{t}^{\prime}\right)^{2}}{s}}$, with $C, b>0$ constant, where the equivalent $|g|_{t}$ moduli are defined in [52]. Locally these moduli are equivalent (so locally there exists a $c>0: c^{-1}|a|_{t} \leq\left|\exp _{t}(a)\right|_{t}^{\prime} \leq c|a|_{t}$, later we will see $c \approx 1$ ) to the weighted modulus on the Lie algebra, see [52, Prop.6.1], which is given by

$$
|a|_{t}=\left|\sum_{i=1}^{3} \beta_{t}^{i} A_{i}^{t}\right|_{t}=\sqrt{\left(\beta_{t}^{1}\right)^{2 / w_{1}}+\left(\beta_{t}^{2}\right)^{2 / w_{2}}+\left|\beta_{t}^{3}\right|^{2 / w_{3}}}
$$

and as a result (derivations will follow below) we have for, respectively, $t \downarrow 0$ and for $t=1$ :

$$
\begin{aligned}
& \bar{K}_{s}^{D_{11}, D_{22}}\left(x, y, e^{i \theta}\right) \leq \frac{1}{4 \pi s^{2} D_{11} D_{22}} e^{-\frac{1}{4 s c^{2}}\left(\frac{x^{2}}{D_{22}}+\frac{\theta^{2}}{D_{11}}+\frac{\left|y-\frac{x \theta}{2}\right|}{\sqrt{D_{11} D_{22}}}\right)}, \\
& K_{s}^{D_{11}, D_{22}}\left(x, y, e^{i \theta}\right) \leq \frac{1}{4 \pi s^{2} D_{11} D_{22}} e^{-\frac{1}{4 s c^{2}}\left(\frac{\theta^{2}}{D_{11}}+\frac{\theta^{2}(y-\eta)^{2}}{4(1-\cos (\theta))^{2} D_{22}}+\frac{1}{\sqrt{D_{11} D_{22}}}\left|\frac{\theta(\xi-x)}{2(1-\cos \theta)}\right|\right)} .
\end{aligned}
$$

A problem though with these estimates is that they are, in contrast to the corresponding exact kernels, not differentiable at, respectively, the surface given by $y=\frac{1}{2} x \theta$ and the surface given by $\tan \theta=\frac{y}{x}$. This is for example a practical problem in generalizing fast regularized derivatives on orientation scores, [30, ch: 5], to the case $D_{22} \neq D_{33}$. This problem can be resolved by applying the estimate

$$
|a|+|b| \geq \sqrt{a^{2}+b^{2}} \geq \frac{1}{\sqrt{2}}(|a|+|b|),
$$


which holds for all $a, b \in \mathbb{R}$, to the exponents of our Gaussian estimates. Therefore we shall estimate the weighted modulus (5.25) by the equivalent (for all $t \geq 0$ ) weighted modulus $|\cdot|^{t}: T_{e}\left((S E(2))_{t}\right) \rightarrow \mathbb{R}^{+}$given by $\left|\sum_{i=1}^{3} \beta_{t}^{i} A_{i}^{t}\right|^{t}:=\sqrt[4]{\left(\left|\beta_{t}^{1}\right|^{2}+\left|\beta_{t}^{2}\right|^{2}\right)^{2}+\left|\beta_{t}^{3}\right|^{2}}$, again indexed by $t \geq 0$. This yields, with again $\xi=x \cos \theta+y \sin \theta, \eta=-x \sin \theta+y \cos \theta$,

$$
\begin{aligned}
& \bar{K}_{s}^{D_{11}, D_{22}}\left(x, y, e^{i \theta}\right) \leq \frac{1}{4 \pi s^{2} D_{11} D_{22}} e^{-\frac{1}{4 s c^{2}} \sqrt{\left(\frac{x^{2}}{D_{22}}+\frac{\theta^{2}}{D_{11}}\right)^{2}+\frac{\left|y-\frac{x \theta}{2}\right|^{2}}{D_{11} D_{22}}}} \\
& K_{s}^{D_{11}, D_{22}}\left(x, y, e^{i \theta}\right) \leq \frac{1}{4 \pi s^{2} D_{11} D_{22}} e^{-\frac{1}{4 s c^{2}} \sqrt{\left(\frac{\theta^{2}}{D_{11}}+\frac{\theta^{2}(y-\eta)^{2}}{4(1-\cos (\theta))^{2} D_{22}}\right)^{2}+\frac{1}{D_{11} D_{22}}\left(\frac{\theta^{2}(\xi-x)^{2}}{4(1-\cos \theta)^{2}}\right)}}
\end{aligned}
$$

where the right-hand sides turn out to be useful asymptotic formulas 10 for the exact Green's functions, in the sense that almost similar lower bounds exist. The latter estimate coincides only locally with the local upper estimate reported by Citti and Sarti [10, Thm. 5.1, eq. 12], $K_{s}^{D_{11}, D_{22}}(g) \leq \frac{C_{1}}{s^{2}} e^{-C_{2}\left(\frac{\xi^{2}}{D_{22}}+\frac{\theta^{2}}{D_{11}}+\frac{|\eta|}{\sqrt{D_{11} D_{22}}}+2 \sqrt{\frac{|\eta|}{\sqrt{D_{11} D_{22}}}} \sqrt{\frac{\xi^{2}}{D_{22}}+\frac{\theta^{2}}{D_{11}}}\right) \frac{1}{4 s}}$, with $g=\left(x, y, e^{i \theta}\right)$ for $|g|_{t}^{\prime}<1$ and some $C_{1}, C_{2}>0$. Our estimate appears to be a much sharper estimate (away from the origin $e$ ). Moreover, the formula by Citti and Sarti is intended as a local upper estimate. It can only be used as a rough approximation of the true kernel close to the unity element if the last term in the exponent is dropped, but even then the restriction of exact kernels to fixed $\theta$ strongly deviates from the exact kernels as $|\theta|$ increases, which is not the case with our asymptotic formulas (5.28).

The advantage of using the Gaussian estimates (5.28) is that they in practice do not require any inverse Fourier transforms, in contrast to our exact formula for the Green's functions on $S E(2)$ and their local approximations, the Green's functions on $H_{3}$, in Theorem 5.1 (for $D_{22} \neq D_{33}$ ). For $\frac{D_{11}}{D_{22}} \ll 1$ the Heisenberg approximation is close to the exact case, so for these parameter settings we may expect (considering the results concerning the equivalent moduli $|\cdot|_{t}, t \in[0,1]$ in [52]) that the estimate for the exact kernel $K_{s}^{D_{11}, D_{22}}$, see (5.26), is sharp as well. Figure 9 shows how the asymptotic formulae (5.28) perform into our scheme of contour enhancement via orientation scores as explained in Section 4 .

5.4.1. Derivation of our estimates by coordinates of the first kind. With respect to the first estimate of (5.26) (and (15.28) $)$ we note that this nilpotent Lie group, which is isomorphic to $H_{3}$, is a subgroup of the five-dimensional group $H_{5}$ of Heisenberg type that arises by approximating $\cos \theta \approx 1$ and $\sin \theta \approx \theta$ with left-invariant vector fields:

$$
\begin{array}{ll}
\hat{A}_{1}=\mathcal{A}_{1}^{0}=\partial_{\theta}, & \hat{A}_{2}=\mathcal{A}_{2}^{0}=\partial_{x}+\theta \partial_{y}, \quad \hat{A}_{4}=\partial_{y}, \\
\hat{A}_{3}=-\theta \partial_{x}+\partial_{y}, & \hat{A}_{5}=\partial_{x} .
\end{array}
$$

This Lie algebra $\mathcal{L}\left(H_{5}\right)=\operatorname{span}\left\{\hat{A}_{1}, \hat{A}_{2}, \hat{A}_{3}, \hat{A}_{4}, \hat{A}_{5}\right\}$ is isomorphic to the matrix algebra

$$
\sum_{i=1}^{5} a^{i} \hat{A}_{i} \leftrightarrow\left(\begin{array}{cccc}
0 & a^{1} & a^{4} & a^{5} \\
0 & 0 & a^{2} & a^{3} \\
0 & 0 & 0 & 0 \\
0 & 0 & 0 & 0
\end{array}\right)=: \sum_{i=1}^{5} a^{i} E_{i}=: B
$$

\footnotetext{
${ }^{10}$ The Gaussian kernels are global upper bounds for the exact $\mathbb{L}_{1}$-normalized positive kernels; therefore they are not $\mathbb{L}_{1}$-normalized. For example, the first Gaussian estimation kernel (5.26) must be multiplied by $\frac{1}{c^{4} 8}$ to be an $\mathbb{L}_{1}$-normalized kernel on $H_{3}$.
} 
The exponent is given by

$$
\exp (t B)=1+t B+\frac{t^{2}}{2} B^{2}=\left(\begin{array}{cccc}
1 & t a^{1} & t a^{4}+\frac{1}{2} t^{2} a^{1} a^{2} & t a^{5}+\frac{1}{2} t^{2} a^{1} a^{3} \\
0 & 1 & t a^{2} & t a^{3} \\
0 & 0 & 1 & 0 \\
0 & 0 & 0 & 1
\end{array}\right) .
$$

This isomorphism enables us to relate the coordinates of the first kind to the coordinates of the second kind in $H_{3}=(S E(2))_{0}$ without explicit use of the CBH-formula :

$$
\begin{aligned}
& (x, y, \theta)=\exp _{0}\left(\alpha^{3} A_{3}\right) \exp _{0}\left(\alpha^{2} A_{2}\right) \exp _{0}\left(\alpha^{1} A_{1}\right)=\exp _{0}\left(\beta_{0}^{1} A_{1}+\beta_{0}^{2} A_{2}+\beta_{0}^{3} A_{3}\right) \Leftrightarrow \\
& \beta_{0}^{1}=\alpha^{1}=\theta, \beta_{0}^{3}+\frac{1}{2} \beta_{0}^{1} \beta_{0}^{2}=\alpha^{3}=y, \beta^{2}=\alpha^{2}=x,
\end{aligned}
$$

with $A_{i}=\left.\hat{A}_{i}\right|_{e} \in T_{e}\left(H_{3}\right), i=1,2,3$. So the coordinates of the first kind on $(S E(2))_{0}$ are

$$
\beta_{0}^{1}=\theta, \beta_{0}^{2}=x \text { and } \beta_{0}^{3}=y-\frac{1}{2} x \theta,
$$

and thereby the weighted modulus on $(S E(2))_{0}$ associated to our filtration (5.22) equals

$$
|g|_{0}=\sqrt{\theta^{2}+x^{2}+\left|y-\frac{1}{2} x \theta\right|} .
$$

Finally we note that the estimate for the Heisenberg approximation (5.26) is reasonably sharp if we relate it to our fundamental solution (5.16) :

$$
\begin{aligned}
& \frac{1}{4 \pi D_{11} D_{22}} \frac{1}{\frac{x^{2}}{D_{22}}+\frac{\theta^{2}}{D_{11}}+\frac{\left|y-\frac{1}{2} x \theta\right|}{\sqrt{D_{11} D_{22}}}} \leq \int_{0}^{\infty} \bar{K}_{s}^{D_{11}, D_{22}}(x, y, \theta) \mathrm{d} s=\frac{1}{\pi D_{11} D_{22}} \frac{1}{\sqrt{\left(\frac{x^{2}}{D_{22}}+\frac{\theta^{2}}{D_{11}}\right)^{2}+16 \frac{\left|y-\frac{1}{2} x \theta\right|^{2}}{D_{11} D_{22}}}} \\
& \quad \leq \frac{1}{\pi D_{11} D_{22}} \frac{x^{2}}{D_{22}+\frac{\theta^{2}}{D_{11}}+\frac{\left|y-\frac{1}{2} x \theta\right|}{\sqrt{D_{11} D_{22}}}}
\end{aligned}
$$

where we recall (5.27). Then we see that $c=\sqrt[4]{2} \approx 1.19>1$ indeed yields a Gaussian upper bound for the exact Heisenberg kernel for $\alpha \downarrow 0$, whereas $c=0.5$ yields a Gaussian lower bound for the same kernel.

Similarly we can derive the second estimate of (5.26) (and (5.28)). To this end we use the formula for exponential curves [20, eq. 3.7] to solve for

$$
\exp \left(\beta_{1}^{1} A_{1}+\beta_{1}^{2} A_{2}+\beta_{1}^{3} A_{3}\right)=\left(x, y, e^{i \theta}\right)
$$

which yields by straightforward computation (for $\theta \in(-\pi, \pi) \backslash\{0\}$ ) that

$$
\beta_{1}^{1}=\theta, \beta_{1}^{2}=\frac{y \theta-\theta \eta}{2(1-\cos \theta)} \text { and } \beta_{1}^{3}=\frac{-x \theta+\xi \theta}{2(1-\cos \theta)},
$$

from which result (5.28) follows. Note that $\frac{\theta}{2(1-\cos \theta)}=\frac{\frac{\theta}{2}}{2 \sin ^{2}\left(\frac{\theta}{2}\right)}$, so again the first-order expansion of $\theta$ in (5.31) gives (5.29) and if $\theta \rightarrow 0$, then $\beta_{t}^{1} \rightarrow 0, \beta_{t}^{2} \rightarrow x, \beta_{t}^{2} \rightarrow y$ for all $t \geq 0$. 


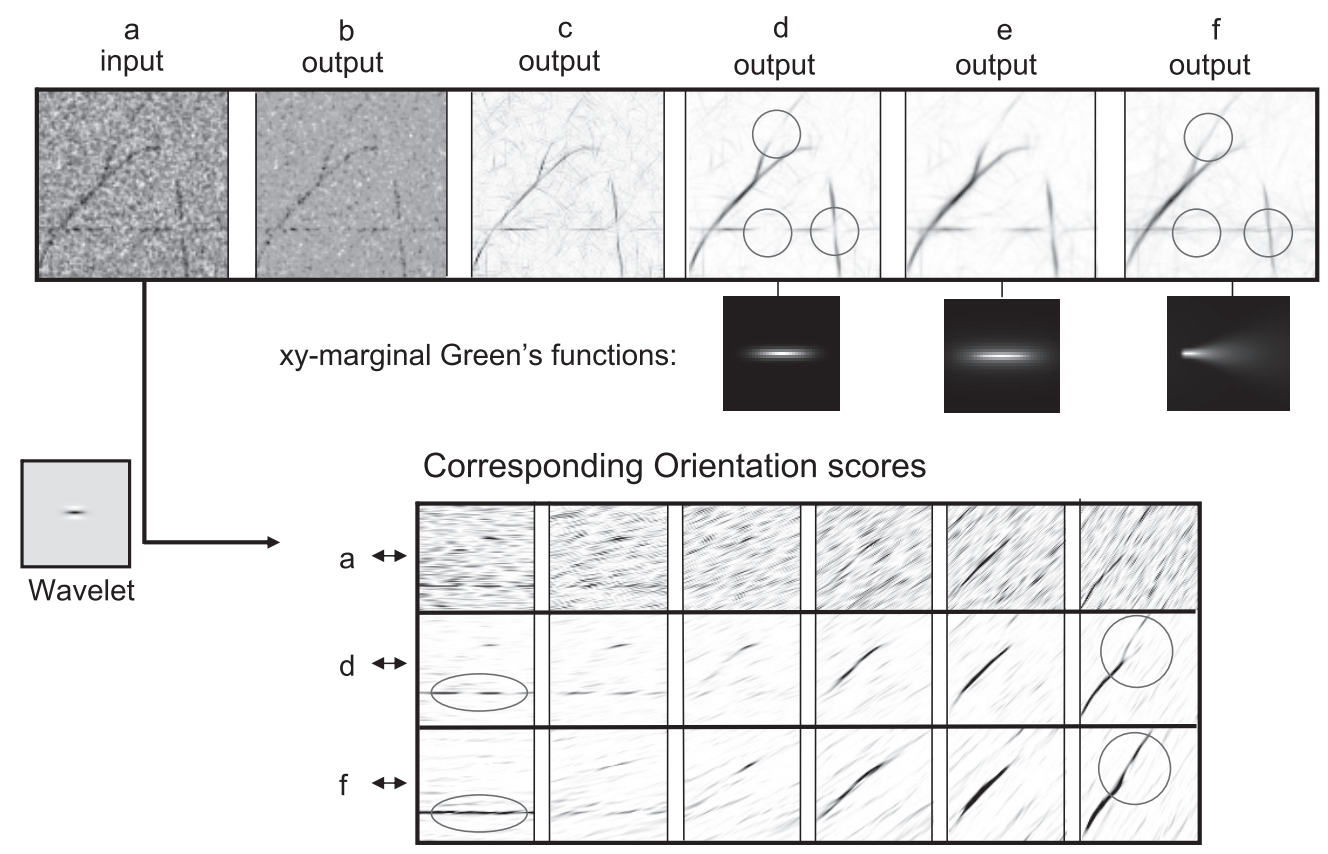

FIG. 9. Top row: From left to right, a: original noisy image $f$, b: $|f|^{p} \operatorname{sign} f$, c: $\left(\mathcal{W}_{\psi}^{*}\right)^{\text {ext }}\left(\chi_{p}\left(\mathcal{W}_{\psi} f\right)\right)$, where we recall that $\chi(U)(x, y, \theta)=|\Re(U(x, y, \theta))|^{p} \operatorname{sign}\{U(x, y, \theta)\}$, d: contour enhancement (4.3) but using time-dependent diffusion kernel depicted below, e: contour enhancement (4.3) using resolvent Green's functions depicted below, f: contour completion using resolvent completion kernel depicted below. In all cases we have set $p=\frac{3}{2}$ and the involved orientation scores are sampled on a $100 \times 100$ grid, using 64 orientations. Circles depict parts of the output images $\Upsilon_{\psi}(f)$, where a clear difference arises in the contour-completion and contour-enhancement processes. Middle row: From left to right, (real part of) proper wavelet $\psi$, where we used the proper wavelets as described in 29. (parameters $q=8, k=2, n_{\theta}=64, t=100, s=20$ ), Green's function time-dependent contour-enhancement process with parameters $D_{11}=0.00015, D_{22}=1$, stopping time $s_{\text {end }}=15$, where we used the asymptotic formula (5.28), Green's function resolvent contourenhancement process $D_{11}=0.00015, D_{33}=1, \alpha=\frac{1}{64}$, Green's function resolvent contour-completion process $D_{11}=0.0024, \alpha=\frac{1}{64}$. Bottom row slices $\mathcal{W}_{\psi} f\left(\cdot, \cdot, e^{i \theta_{k}}\right)$ for $\theta_{k}=(2 k+1) \frac{\pi}{32}, k, 0,1,2,3,4,5$, in the corresponding orientation scores of the output image.

Appendix A. Invertibility of transforms $\mathcal{W}_{\psi}$, resp. $W_{\psi}$, and decomposition of $\mathcal{V}$, resp. $\mathcal{U}$, into irreducible representations and an inverse Fourier transform on $S E(2)$, resp. $S I M(2)$. So far we have presented a summary of results (relevant for applications) of our previous theory on invertible orientation scores. A natural question that arises to the reader unfamiliar with the previous works [14, 31], 33], [3] is how does the invertibility of the transform $W_{\psi}$, given by

$$
\left(\mathbb{R}^{2} \ni(x, y) \mapsto f(x, y) \in \mathbb{R}\right) \rightarrow\left(S I M(2) \ni g \mapsto W_{\psi} f(g)=\left(\mathcal{V}_{g} \psi, f\right) \in \mathbb{C}\right),
$$


relate to the irreducibility of the underlying representation $\mathcal{V}$; recall (2.3). Secondly, the question arises how this relates to the fact that we must set $M_{\psi}=1$ (recall (2.7) and (2.6) ) to guarantee well-posed reconstruction of the transform $\mathcal{W}_{\psi}$, given by

$$
\left(\mathbb{R}^{2} \ni(x, y) \mapsto f(x, y) \in \mathbb{R}\right) \rightarrow\left(S E(2) \ni g \mapsto \mathcal{W}_{\psi} f(g)=\left(\mathcal{U}_{g} \psi, f\right) \in \mathbb{C}\right),
$$

by means of its $\mathbb{L}_{2}$-adjoint $\left(\mathcal{W}_{\psi}\right)^{*}$. In this section we provide a brief answer to these important questions.

Although the early works of Grossmann et al., 33, 3. are mostly based on decomposition of the identity using extended versions of Schur's lemma, an alternative and shorter answer to this question can be deduced from [31, ch:4] by exploiting the relation between the transforms $W_{\psi}: \mathbb{L}_{2}\left(\mathbb{R}^{2}\right) \rightarrow \mathbb{L}_{2}(S I M(2))(2.2)$ and $\mathcal{W}_{\psi}: \mathbb{L}_{2}\left(\mathbb{R}^{2}\right) \rightarrow \mathbb{L}_{2}(S E(2))$ (1.1) and inverse Fourier transforms on, respectively, the groups $S I M(2)$ and $S E(2)$. However, since the important and general work in [31] is rather abstract, we will focus only on our specific cases of interest and provide the explicit formulae for these cases.

To this end we use the general identity: $\operatorname{trace}\left\{a \otimes b \circ A^{*}\right\}=(A b, a)$, where $A$ is some bounded linear operator on a Hilbert space and $a$ and $b$ some vectors in the Hilbert space, where we define $(a \otimes b)(x)=(b, x) a$. As a result we rewrite the wavelet transform $W_{\psi}$ as

$$
\begin{aligned}
W_{\psi} f(g) & =\left(\mathcal{V}_{g} \psi, f\right)_{\mathbb{L}_{2}\left(\mathbb{R}^{2}\right)}=\operatorname{trace}\left\{f \otimes \psi \circ\left(\mathcal{V}_{g}\right)^{*}\right\}=\int_{\widehat{S I M}(2)} \operatorname{trace}\left\{A_{f, \psi}(\sigma) \sigma\left(g^{-1}\right)\right\} \frac{\mathrm{d} \nu \widehat{S I M}(2)}{\nu_{\overparen{S I M}(2)}(\sigma)} \\
& =\frac{1}{\nu_{\overparen{S I M}(2)}(\mathcal{V})}\left[\mathcal{F}_{S I M(2)}^{-1}\left(A_{f, \psi}\right)\right]\left(g^{-1}\right)
\end{aligned}
$$

where $\nu_{\widehat{S I M}(2)}$ denotes the Plancherel measure on the dual group $\widehat{S I M}(2)$, [27], consisting of all nonequivalent, unitary, irreducible representations of the group $S I M(2)$ and where

$$
A_{f, \psi}(\sigma)= \begin{cases}0 & \text { if } \sigma \neq \mathcal{V} \\ f \otimes \psi & \text { if } \sigma=\mathcal{V}\end{cases}
$$

So the Plancherel theorem for the Fourier transform on the group $S I M(2)$ now yields

$$
\left\|\mathcal{W}_{\psi} f\right\|_{\mathbb{L}_{2}(S I M(2))}^{2}=\left.\int_{\widehat{S I M}(2)}\left\|A_{f, \psi}(\sigma)\right\|\right|^{2} \frac{\mathrm{d} \nu_{\widehat{S I M}(2)}(\sigma)}{\nu \widehat{\operatorname{SIM}(2)}(\mathcal{V})}=\|f\|_{\mathbb{L}_{2}\left(\mathbb{R}^{2}\right)}^{2}\|\psi\|_{\mathbb{L}_{2}\left(\mathbb{R}^{2}\right)}^{2} \frac{1}{\nu_{\overline{S I M}(2)}(\mathcal{V})}
$$

where $\||\cdot|||$ denotes the Hilbert-Schmidt norm, which is defined on bounded operators $A \in \mathcal{B}\left(\mathbb{L}_{2}\left(\mathbb{R}^{2}\right)\right)$ acting on $\mathbb{L}_{2}\left(\mathbb{R}^{2}\right)$ by means of

$$
\|A\|\left\|^{2}=\operatorname{trace}\left\{A^{*} A\right\}=\sum_{k=1}^{\infty}\right\| A f_{k} \|^{2}, \text { where }\left\{f_{k}\right\}_{k=1}^{\infty} \text { is some orthonormal basis for } \mathbb{L}_{2}\left(\mathbb{R}^{2}\right),
$$

so in particular, the Hilbert-Schmidt norm of the tensor product $f \otimes \psi$ of $\psi$ and $f$ equals $\|f \otimes \psi\|\left\|^{2}=\right\| f\left\|^{2}\right\| \psi \|^{2}$. We conclude that the admissibility constant (2.4) equals $C_{\psi}=$ $\frac{1}{\nu_{\overparen{S I M}(2)}(\mathcal{V})}\|\psi\|^{2}$ and, moreover, the unitarity of $\mathcal{W}_{\psi}$ directly follows from the Plancherel theorem on $\operatorname{SIM}(2)$ and the fact that $\mathcal{V} \in \widehat{S I M}(2)$.

Obviously, one would like to obtain the same kind of connection between the transform $\mathcal{W}_{\psi}: \mathbb{L}_{2}\left(\mathbb{R}^{2}\right) \rightarrow \mathbb{L}_{2}(S E(2))$ and the inverse Fourier transform on $S E(2)$, but here arises a technical problem. In contrast to the representation $\mathcal{V}$, the representation $\mathcal{U}$ is reducible. Therefore it must be decomposed into irreducible representations; i.e., $\mathcal{U}$ must 
be written as a direct integral of irreducible representations. This is similar to the wellknown Peter-Weyl theorem for compact groups, [50, but the technical problem is that $S E(2)$ is not compact, giving rise to an over-countable set of irreducible representations requiring direct integral decomposition (for details on these decompositions, see [31, pp. 67-84]) rather than direct sum decomposition. All unitary, irreducible representations, up to unitary equivalence, of $S E(2)$ are given in [51] and the ones with nontrivial dual Plancherel measure occur only once in the direct integral decomposition of $\mathcal{U}$. They can be related to the dual orbits of $S O(2)$ on $\mathbb{R}^{2}$ which coincide with rings in the Fourier domain, using Mackey's theory 42. Now the theoretical rationale behind $M_{\psi}=1$, recall (2.6), is that the kernel $\psi$ must be chosen with unit length in each irreducible subspace of $\mathbb{L}_{2}\left(\mathbb{R}^{2}\right) \cap \mathbb{L}_{1}\left(\mathbb{R}^{2}\right)$, meaning that the $\mathbb{L}_{2}$-norm over each fixed ring in the Fourier domain is 1 (note that $M_{\psi}(\boldsymbol{\omega})$ only depends on the radius $\rho=\|\boldsymbol{\omega}\|$ ) so that each irreducible subspace of $\mathbb{L}_{2}\left(\mathbb{R}^{2}\right) \cap \mathbb{L}_{1}\left(\mathbb{R}^{2}\right)$ is unitarily mapped to each irreducible subspace of the space of orientation scores $\mathcal{R}\left(\mathcal{W}_{\psi}\right) \subset \mathbb{L}_{2}(S E(2))$.

Let us verify these statements on both the transform $\mathcal{W}_{\psi}$ between images and orientation scores and the corresponding reducible representation $\mathcal{U}$ by explicit formulas. First of all we define the representations $\tilde{\mathcal{U}}^{\rho}: S E(2) \rightarrow \mathcal{B}\left(\mathbb{L}_{2}\left(S_{\rho}\right)\right)$, where $\mathcal{B}\left(\mathbb{L}_{2}\left(S_{\rho}\right)\right)$ stands for all bounded operators on the space $\mathbb{L}_{2}\left(S_{\rho}\right)$ of quadratic integrable function(s) (classes) defined on the sphere $S_{\rho}=\left\{\boldsymbol{\omega} \in \mathbb{R}^{2} \mid\|\boldsymbol{\omega}\|=\rho\right\}$, given by

$$
\tilde{\mathcal{U}}_{g}^{\rho} F(\rho \cos \varphi, \rho \sin \varphi)=e^{i(\rho \cos \varphi, \rho \sin \varphi) \cdot(x, y)} F(\rho \cos (\varphi-\theta), \rho \sin (\varphi-\theta)),
$$

for all $g=\left(x, y, e^{i \theta}\right) \in S E(2), F \in \mathbb{L}_{2}\left(S_{\rho}\right)$. These representations are unitarily equivalent to well-known unitary, irreducible representations of $S E(2)$, [51, 9, ch: 10.2], given by

$$
\mathcal{U}_{g}^{\rho} \phi(\mathbf{v})=e^{-i \rho(x, v)} \phi\left(\left(R_{\theta}\right)^{-1} \mathbf{v}\right), \quad \rho>0, \phi \in \mathbb{L}_{2}\left(S_{1}\right), \mathbf{v} \in S_{1}, g=\left(\mathbf{x}, e^{i \theta}\right) \in S E(2),
$$

since $\tilde{\mathcal{U}}_{g}^{\rho}=\mathcal{D}_{\rho} \circ \mathcal{U}_{g}^{\rho} \circ \mathcal{D}_{\rho}^{-1}$, where the dilation operator $\mathcal{D}_{\rho}: \mathbb{L}_{2}\left(S_{1}\right) \rightarrow \mathbb{L}_{2}\left(S_{\rho}\right)$ is unitary and $\mathcal{D}_{\rho} \phi(\mathbf{v})=\rho^{-\frac{1}{2}} \phi\left(\rho^{-1} \mathbf{v}\right)$.

Consider the dual orbit space $\mathbb{T} \backslash \mathbb{R}^{2}$, where the dual orbits are given by $S_{\rho}=\left\{A^{T} \boldsymbol{\omega} \mid\right.$ $A \in S O(2)\}$, with $\rho=\|\boldsymbol{\omega}\|$. Then we have the following direct integral decomposition:

$$
\mathcal{F} \circ \mathcal{U}_{g} \circ \mathcal{F}^{-1}=\int_{\mathbb{R}^{+} \equiv \mathbb{T}_{\mathbb{R}}}^{\oplus} \tilde{\mathcal{U}}_{g}^{\rho} \mathrm{d} \nu\left(S_{\rho}\right),
$$

where the measure on the dual orbits by identification $\rho \in \mathbb{R}^{+} \equiv S_{\rho} \in \mathbb{T} \backslash \mathbb{R}^{2}$ equals $\mathrm{d} \nu\left(S_{\rho}\right)=\rho \mathrm{d} \rho$. Analogously to (A.1), we have

$$
\begin{aligned}
& \mathcal{W}_{\psi} f(g)=\left(\mathcal{U}_{g} \psi, f\right)_{\mathbb{L}_{2}\left(\mathbb{R}^{2}\right)}=\left(\int_{\mathbb{R}^{+}}^{\oplus} \tilde{\mathcal{U}}_{g}^{\rho} \rho \mathrm{d} \rho \mathcal{F} \psi, \mathcal{F} f\right)_{\mathbb{L}_{2}\left(\mathbb{R}^{2}\right)} \\
& =\int_{0}^{\infty}\left(\left.\left(\tilde{\mathcal{U}}_{g}^{\rho}\right) \mathcal{F} \psi\right|_{S_{\rho}},\left.\mathcal{F} f\right|_{S_{\rho}}\right) \rho \mathrm{d} \rho=\int_{0}^{\infty} \operatorname{trace}\left(\left(\left.\left.\mathcal{F} f\right|_{S_{\rho}} \otimes \mathcal{F} \psi\right|_{S_{\rho}}\right) \circ \tilde{\mathcal{U}}_{g^{-1}}^{\rho}\right) \rho \mathrm{d} \rho .
\end{aligned}
$$

Now $\rho \mapsto \tilde{\mathcal{U}}^{\rho}$ is injective into the dual group $\widehat{S E}(2)$, since $\tilde{\mathcal{U}}^{\rho}$ is unitarily equivalent to the unitary irreducible representations (A.2). Moreover $\mathrm{d} \nu\left(S_{\rho}\right)$ equals the restriction of the Plancherel measure to $\left\{\tilde{\mathcal{U}}^{\rho}\right\}_{\rho>0}$, 51, so we see that (A.3) can be rewritten as

$$
\mathcal{W}_{\psi} f\left(g^{-1}\right)=\mathcal{F}_{S E(2)}^{-1}\left(\left.\left.\rho \mapsto \mathcal{F} f\right|_{S_{\rho}} \otimes \mathcal{F} \psi\right|_{S_{\rho}}\right)(g) .
$$


Now by the Plancherel theorem on both $S E(2)$, [51, 9, and $\mathbb{R}^{2}$ one has

$$
\begin{aligned}
& \left\|\mathcal{W}_{\psi} f\right\|_{\mathbb{L}_{2}(S E(2))}^{2}=\left.\int_{0}^{\infty}\left\||\mathcal{F} f|_{S_{\rho}}\right\|\right|^{2} \rho \mathrm{d} \rho=\int_{0}^{\infty}\left\|\left.\mathcal{F} \psi\right|_{S_{\rho}}\right\|_{\mathbb{L}_{2}\left(S_{\rho}\right)}^{2}\left\|\left.\mathcal{F} f\right|_{S_{\rho}}\right\|_{\mathbb{L}_{2}\left(S_{\rho}\right)}^{2} \rho \mathrm{d} \rho, \\
& \|f\|_{\mathbb{L}_{2}\left(\mathbb{R}^{2}\right)}^{2}=\|\mathcal{F} f\|_{\mathbb{L}_{2}\left(\mathbb{R}^{2}\right)}^{2}=\int_{0}^{\infty}\left\|\left.\mathcal{F} f\right|_{S_{\rho}}\right\|_{\mathbb{L}_{2}\left(S_{\rho}\right)}^{2} \rho \mathrm{d} \rho,
\end{aligned}
$$

so indeed we have the following sufficient and necessary condition for $\mathbb{L}_{2}$-norm preservation:

$$
M_{\psi}=1 \text { i.e. }\left\|\left.\mathcal{F} \psi\right|_{S_{\rho}}\right\|_{\mathbb{L}_{2}\left(S_{\rho}\right)}^{2}=1 \text { for all } \rho=\|\boldsymbol{\omega}\|>0,
$$

where we recall the definition of $M_{\psi}$ (2.7) and where we note that $M_{\psi}(\boldsymbol{\omega})=$ $\left\|\left.\mathcal{F} \psi\right|_{S_{\rho=\|} \boldsymbol{\omega}_{\|}}\right\|_{\mathbb{L}_{2}\left(S_{\rho}\right)}^{2}$. Moreover in the case $M_{\psi}=1$, for $\psi \in \mathbb{L}_{2}\left(\mathbb{R}^{2}\right) \cap \mathbb{L}_{1}\left(\mathbb{R}^{2}\right)$, its continuous Fourier transform $\mathcal{F} \psi$ has equal $\mathbb{L}_{2}$-norm over each dual orbit, implying that each irreducible subspace of $\mathbb{L}_{2}\left(\mathbb{R}^{2}\right) \cap \mathbb{L}_{1}\left(\mathbb{R}^{2}\right)$ given by $\left\{f \in \mathbb{L}_{2}\left(\mathbb{R}^{2}\right) \cap \mathbb{L}_{1}\left(\mathbb{R}^{2}\right) \mid \operatorname{supp} \mathcal{F} f \subset S_{\rho}\right\}$ is unitarily mapped onto each irreducible subspace within $\mathcal{R}\left(\mathcal{W}_{\psi}\right) \subset \mathbb{L}_{2}(S E(2))$.

Acknowledgements. The authors are grateful to the reviewer for his good suggestions on the organization and formulation of this paper. Moreover, the authors gratefully acknowledge his thoughtful comments on the contents of earlier drafts. They also express their thanks to David Mumford, Brown University, USA, for his encouragement in the course of the work leading up to the paper. Moreover, the authors are grateful to Prof. Hartmut Führ, from RWTH Aachen, Germany, for pointing us to the fact that it is easier to relate wavelet transforms to inverse Fourier transform, Appendix A than to relate inverse wavelet transforms to Fourier transform.

\section{REFERENCES}

[1] M. Abramowitz and I. A. Stegun, editors. Handbook of Mathematical Functions with Formulas, Graphs, and Mathematical Tables. Dover Publications, Inc., New York, 1965. Originally published by the National Bureau of Standards in 1964. MR0167642 (29:4914)

[2] M. A. van Almsick. Context Models of Lines and Contours, Ph.D. thesis, Eindhoven University of Technology, Department of Biomedical Engineering, Eindhoven, the Netherlands, 2007.

[3] S.T. Ali, J.P. Antoine and J.P. Gazeau. Coherent States, Wavelets and Their Generalizations. Springer-Verlag, New York, Berlin, Heidelberg, 1999 MR.1735075 (2002m:81092)

[4] J.P. Antoine. Directional wavelets revisited: Cauchy wavelets and symmetry detection in patterns. Applied and Computational Harmonic Analysis, 6:314-345, 1999. MR.1685408 (2000b:42025)

[5] N. Aronszajn. Theory of reproducing kernels. Trans. Amer. Math. Soc., vol. 68, pp.337-404, 1950. MR0051437(14:479c)

[6] J. August. The Curve Indicator Random Field. Ph.D. thesis, Yale University, 2001.

[7] J. August and S.W. Zucker. The curve indicator random field and Markov processes. IEEE-PAMI, Pattern Recognition and Machine Intelligence, 25, 2003. Number 4.

[8] W.H. Bosking, Y. Zhang, B. Schofield, and D. Fitzpatrick. Orientation selectivity and the arrangement of horizontal connections in tree shrew striate cortex. The Journal of Neuroscience, 17(6):2112-2127, March 1997.

[9] G.S. Chirikjian and A.B. Kyatkin. Engineering applications of non-commutative harmonic analysis with emphasis on rotation and motion groups. CRC Press LLC, USA, 2001. MR1885369 (2003g:42001)

[10] G. Citti and A. Sarti. A cortical based model of perceptional completion in the roto-translation space. Journal of Mathematical Imaging and Vision, 24 (3):307-326, 2006. MR2235475 (2007b:92016)

[11] A. V. Bukhvalov and W. Arendt. Integral representations of resolvents and semigroups. Forum Math. 6, 6(1):111-137, 1994. MR1253180 (94k:47062) 
[12] M. Duits. A functional Hilbert space approach to frame transforms and wavelet transforms. September 2004. Master thesis in Applied Analysis. Dept. Mathematics and Computer Science, Eindhoven University of Technology.

[13] M. Duits and R. Duits. Unitary wavelet transforms based on reducible representations of the affine group. In preparation.

[14] R. Duits. Perceptual Organization in Image Analysis. Ph.D. thesis, Eindhoven University of Technology, Department of Biomedical Engineering, The Netherlands, 2005. A digital version is available on the web URL: http:// www.bmi2.bmt.tue.nl/ImageAnalysis/People/RDuits/THESISRDUITS.pdf.

[15] R. Duits, M. Felsberg, G. Granlund, and B.M. ter Haar Romeny. Image analysis and reconstruction using a wavelet transform constructed from a reducible representation of the Euclidean motion group. International Journal of Computer Vision. Volume 72, issue 1, p.79-102, April 2007.

[16] R. Duits, M. Duits, M. van Almsick and B.M. ter Haar Romeny. Invertible Orientation Scores as an Application of Generalized Wavelet Theory. Image Processing, Analysis, Recognition, and Understanding. Volume 17, Nr. 1: p. 42-75, 2007.

[17] R. Duits, L.M.J. Florack, J. de Graaf, and B. ter Haar Romeny. On the axioms of scale space theory. Journal of Mathematical Imaging and Vision, 20:267-298, May 2004. MR2060148 (2005k:94005)

[18] R. Duits, M. van Almsick, M. Duits, E. Franken, and L.M.J. Florack. Image processing via shifttwist invariant operations on orientation bundle functions. In Niemann Zhuralev et al. Geppener, Gurevich, editor, 7th International Conference on Pattern Recognition and Image Analysis: New Information Technologies, pages 193-196, St. Petersburg, October 2004.

[19] R. Duits and B. Burgeth. Scale Spaces on Lie groups Proceedings, of SSVM 2007, 1st international conference on scale space and variational methods in computer vision, Lecture Notes on Computer Science, pp. 300-312, editors: Sgallari, Murli and Paragios, Springer-Verlag, June 2007.

[20] R. Duits and M. van Almsick. The Explicit Solutions of Linear Left-Invariant Second Order Stochastic Evolution Equations on the 2D Euclidean Motion Group. Quarterly of Applied Mathematics, Vol. 66, pp. 27-67, April 2008. MR2396651

[21] R. Duits and E.M. Franken. Left-invariant Stochastic Evolution Equations on $S E(2)$ and its Applications to Contour Enhancement and Contour Completion via Invertible Orientation Scores. arXiv: 0711.0951v4, see http://arxiv.org/abs/0711.0951. Also available as CASA report no. 35, 2007, Eindhoven University of Technology, http://www.win.tue.nl/casa/research/casareports/2007.html

[22] R. Duits and E.M. Franken. Left-invariant Parabolic Evolutions on SE(2) and Contour Enhancement via Invertible Orientation Scores, Part II: Nonlinear left-invariant diffusions on invertible orientation scores. Quarterly of Applied Mathematics, this volume.

[23] N. Dungey, A. F. M. ter Elst, and D. W. Robinson. Analysis on Lie groups with polynomial growth, volume 214. Progress in Mathematics, Birkhäuser, Boston, 2003. MR2000440 (2004i:22010)

[24] M. Felsberg, P.-E. Forssén, and H. Scharr. Channel smoothing: Efficient robust smoothing of lowlevel signal features. IEEE Transactions on Pattern Analysis and Machine Intelligence, vol. 28 : no. 2: p. 209-202, 2006.

[25] G. Floquet. Sur les équations différentielles linéaires à coefficients périodiques. Ann. Sci. École Norm. Sup., 12, 1883, pp. 47-88. MR.1508722

[26] L.M.J. Florack. Image Structure. CIV, Vol. 10, Kluwer Academic Publishers Dordrecht, The Netherlands, 1997.

[27] G.B. Folland. A Course in Abstract Harmonic Analysis. Studies in Advanced Mathematics, CRC Press, 1995. MR:1397028 (98c:43001)

[28] P.E. Forssen. Low and Medium Level Vision using Channel Representations. Ph.D. thesis, Linkoping University, Dept. EE, Linkoping, Sweden, March 2004.

[29] E. Franken, R. Duits and B.M. ter Haar Romeny, Non-linear Diffusion on the Euclidean Motion Group. Proc. of the first international conference on Scale Space and Variational Methods in Computer Vision, 461-472, 2007.

[30] E.M. Franken, Enhancement of Crossing Elongated Structures in Images. Ph.D. thesis, Eindhoven University of Technology, Department of Biomedical Engineering, 2008, Eindhoven, The Netherlands, available on the web, URL: http://www.bmi2.bmt.tue.nl/ImageAnalysis/People/EFranken/PhDThesisErikFranken.pdf .

[31] H. Führ. Abstract Harmonic Analysis of Continuous Wavelet Transforms. Springer, HeidelbergNew York, 2005. MR2130226 (2006m:43003) 
[32] B. Gaveau. Principe de moindre action, propagation de la chaleur et estimées sous elliptiques sur certains groupes nilpotents. Acta Mathematica, 139:96-153, 1977. MR0461589 (57:1574)

[33] A. Grossmann, J. Morlet, and T. Paul. Integral transforms associated to square integrable representations. J. Math. Phys., 26:2473-2479, 1985. MR.803788 (86k:22013)

[34] W. Hebisch. Estimates on the semigroups generated by left-invariant operators on Lie groups. Journal fuer die reine und angewandte Mathematik, 423:1-45, 1992. MR1142482 (93d:22008)

[35] G. W. Hill. On the part of motion of the lunar perigee, which is a function of the mean motions of the sun and the moon. Acta Mathematica, 1, 1886.

[36] L. Hörmander. Hypoellptic second order differential equations. Acta Mathematica, 119:147-171, 1967. MR 0222474 (36:5526)

[37] S. N. Kalitzin, B. M. ter Haar Romeny, and M. A. Viergever. Invertible apertured orientation filters in image analysis. Int. Journal of Computer Vision, 31(2/3):145-158, April 1999.

[38] J. J. Koenderink. The Structure of Images. Biol. Cybernetics, (50), pp. 363-370, 1984. MR758126

[39] T. S. Lee. Image representation using 2 d gabor wavelets. IEEE-Transactions on Pattern Analysis and Machine Intelligence, 18(10):959-971, 1996.

[40] P. Lévy. Wiener Random Functions and other Laplacian Random Functions Proc. of the 2nd Berkeley Symposium. California Press, USA, 171-187, 1950.

[41] A. K. Louis, P. Maass and A. Rieder. Wavelets, Theory and Applications. B. G. Teubner, Stuttgart, 1998. MR $1681147(99 \mathrm{k}: 42063)$

[42] G.W. Mackey. Imprimitivity for representations of locally compact groups Proc. Nat. Acad. Sci. 35: 537-545, 1949. MR0031489 (11:158b)

[43] W. Magnus and S. Winkler. Hill's equation. Dover, New York, 1979. MR559928 (80k:34001)

[44] Gérard Medioni, Mi-Suen Lee, and Chi-Keung Tang. A Computational Framework for Segmentation and Grouping. Elsevier, Amsterdam.

[45] J. Meixner and F. W. Schaefke. Mathieusche Funktionen und Sphaeroidfunktionen. Springer-Verlag, Berlin-Gottingen-Heidelberg, 1954.

[46] F.J.L. Martens. Spaces of analytic functions on inductive/projective limits of Hilbert Spaces. Ph.D. thesis, University of Technology Eindhoven, The Netherlands, Department of Mathematics and Computing Science, 1988, URL: http://alexandria.tue.nl/extra3/proefschrift/PRF6A/8810117.pdf $\operatorname{MR} 971026(90 \mathrm{k}: 46051)$

[47] D. Mumford. Elastica and computer vision. Algebraic Geometry and Its Applications. SpringerVerlag, pages 491-506, 1994. MR1272050 (95a:92026)

[48] B. Øksendahl. Stochastic differential equations: An introduction with applications. Springer, Berlin, 1998. MR1619188 (99c:60119)

[49] N. Otsu. Mathematical Studies on Feature Extraction in Pattern Recognition. Ph.D. thesis, Electrotechnical Laboratory, 818-Ibaraki, Japan, 1981.

[50] W. Schempp and B. Dreseler. Einführung in die Harmonische Analyse. B. G.Teubner, Stuttgart, 1980. MR582460 (82m:43001)

[51] M. Sugiura Unitary representations and harmonic analysis. second edition, North-Holland Mathematical Library, 44, Amsterdam, Kodansha, Tokyo, 1990. MR1049151 (91c:22028)

[52] A.F.M. ter Elst and D.W. Robinson Weighted Subcoercive Operators on Lie Groups. Journal of Functional Analysis, 157:88-163, 1998. MR1637929 (99h:47055)

[53] K.K. Thornber and L.R. Williams. Characterizing the Distribution of Completion Shapes with Corners Using a Mixture of Random Processes. Pattern Recognition, 33:543-553, 2000.

[54] M. A. van Almsick, R. Duits, E. Franken, and B.M. ter Haar Romeny. From stochastic completion fields to tensor voting. In Proceedings DSSCC-workshop on Deep Structure Singularities and Computer Vision, pages 124-134, Springer-Verlag, Maastricht, the Netherlands, June 9-10 2005.

[55] V.S. Varadarajan. Lie Groups, Lie algebras and Their Representations. Springer-Verlag, New York, Berlin, Heidelberg, Tokyo, 1984. MR746308 (85e:22001)

[56] L. R. Williams and J.W. Zweck. A rotation and translation invariant saliency network. Biological Cybernetics, 88:2-10, 2003.

[57] L. R. Williams and D.W. Jacobs. Stochastic Completion Fields: A Neural model of Illusory Contour Shape and Salience. Neural Computation, 9(4), p.837-858, 1997.

[58] O. Wittich. An explicit local uniform large deviation bound for Brownian bridges. Statistics and Probability Letters, 73(1):51-56, 2005. MR2154059 (2006d:60055) 
[59] J. Zweck and L. R. Williams. Euclidean group invariant computation of stochastic completion fields using shiftable-twistable functions. Journal of Mathematical Imaging and Vision, 21(2):135-154, 2004. MR2090129 (2005d:68131) 